\title{
New Mixed Phosphonate Esters by Transesterification of Pinacol- phosphonates and their use in Aldehyde and Ketone Coupling Reactions with Non-stabilized Phosphonates
}

\author{
John F. Reichwein and Brian L. Pagenkopf* \\ Department of Chemistry and Biochemistry, The University of Texas at Austin, \\ Austin, TX 78712
}

\section{Supporting Information}

The final products $\mathbf{1 2 b},{ }^{1} \mathbf{1 2 c},{ }^{2} \mathbf{1 2 d}^{3}$ and $\mathbf{1 2 \mathrm { e } ^ { 4 }}$ are known compounds.

To circumvent the pinacol ester cleavage altogether, other hydrogen phosphites were investigated in the rhodium catalyzed alkene hydrophosphorylation reaction. For this study the hydrogen phosphonites and phosphinic acids in Figure 1 were selected because of their easy preparation (i) ${ }^{5}$ the structural homology they shared with 1 (ii-iii and v), ${ }^{6}$ and the commercial availability of precursors (iv). The hydrogen phosphites were prepared by hydrolysis of commercial chlorophosphines (iv), or by the condensation of $\mathrm{PCl}_{3}$ with the corresponding diols (i-ii), 2-mercaptoethanol (iii) or diamine (v). However, ii-a and iii were useless for hydrophosphorylation because they polymerized within hours of preparation, ${ }^{7}$ ii-b underwent explosive decomposition during purification by vacuum distillation $\left(0.4 \mathrm{~mm} \mathrm{Hg}, \sim 130{ }^{\circ} \mathrm{C}\right){ }^{8}$ and ii-c was insoluble in common organic solvents. ${ }^{6}$ The hydrophosphorylation of octene with the remaining hydrogen phosphonites $\mathbf{i}$, iv, and $\mathbf{v}$ with Wilkinson's catalyst was unsuccessful. A similar lack of reactivity was observed in a palladium catalyzed olefin hydrophosphorylation study with $(\mathrm{MeO})_{2} \mathrm{P}(\mathrm{O}) \mathrm{H}$, $(i-\mathrm{PrO})_{2} \mathrm{P}(\mathrm{O}) \mathrm{H},(\mathrm{PhO})_{2} \mathrm{P}(\mathrm{O}) \mathrm{H}$ and i-b. ${ }^{9}$ Although alkyne hydrophosphorylation has been achieved with $\mathrm{Ph}_{2} \mathrm{P}(\mathrm{O}) \mathrm{H},{ }^{10} \mathrm{Ph}(\mathrm{OMenth}) \mathrm{P}(\mathrm{O}) \mathrm{H},{ }^{11}(\mathrm{MeO})_{2} \mathrm{P}(\mathrm{O}) \mathrm{H}$ and $(\mathrm{EtO})_{2} \mathrm{P}(\mathrm{O}) \mathrm{H}^{12}$ using a palladium catalyst, ${ }^{9}$ and intramolecular hydrophosphination of unactivated olefins has been reported, ${ }^{13,14,15}$ at its current state of development olefin hydrophosphorylation remains restricted to hydrogen phosphite $\mathbf{1}$.

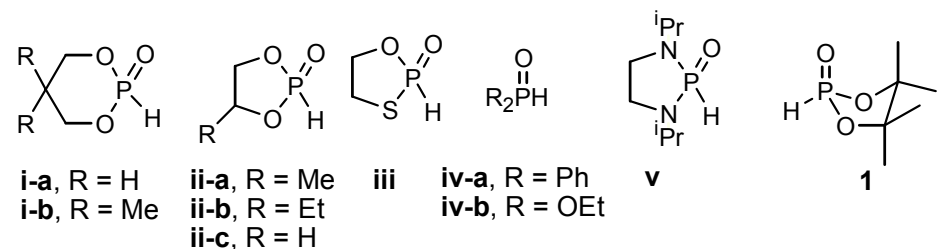

Figure 1. Hydrogen phosphonites screened with $\left(\mathrm{PPh}_{3}\right)_{3} \mathrm{RhCl}$.

\footnotetext{
${ }^{1}$ Halm, C.; Evarts, J.; Kurth, M. J. Tetrahedron Lett. 1997, 38, 7709-7712.

${ }^{2}$ See e.g. Clarke, S. D.; Harrison, C. R.; Hodge, P. Tetrahedron Lett. 1980, 21, 1375-1378.

${ }^{3}$ Barton, D. H. R.; Jaszberenyi, J. C.; Tachdjian, C. Tetrahedron Lett. 1991, 32, 2704-2706.

${ }^{4}$ See e.g. Walker, L. F.; Bourghida, A.; Connolly, S.; Wills, M. J. Chem. Soc., Perkin Trans. 1 2002, 7, 965-981.

${ }^{5}$ Zwierzak, A. Can. J. Chem. 1967, 45, 2501-2512.

${ }^{6}$ Lucas, H. J.; Mitchell, F. W. Jr.; Scully, C. N. J. Am. Chem. Soc. 1950, 72, 5491-5497.

${ }^{7}$ Nifant'ev, E. E.; Nasonovskii, I. S.; Miklashevskii, A. V.; Zavalishina, A. I.; Smirnova, E. I. Zh. Org. Khim. 1975, 11, $2206-2210$.

${ }^{8}$ A similar observation has been reported for ii-a, ref 6

${ }^{9}$ Han, L.-B.; Mirzaei, F.; Zhao, C.-Q.; Tanaka, M. J. Am. Chem. Soc. 2000, 122, 5407-5408.

${ }^{10}$ (a) Han, L.-B.; Choi, N.; Tanaka, M. Organometallics 1996, 15, 3259-3261; (b) Hua, R.; Tanaka, M. Chem. Lett. 1998, 431-432; Han, L.B.; Zhao, C.-Q.; (c) Tanaka, M. J. Org. Chem. 2001, 66, 5929-5932; (d) Allen, A. Jr.; Ma, L.; Lin, W. Tetrahedron Lett. 2002, 43, 37073710 .

${ }^{11}$ Han, L.-B.; Zhao, C.-Q.; Onozawa, S.-Y.; Goto, M.; Tanaka, M. J. Am. Chem. Soc. 2002, 124, 3842-3843.

${ }^{12}$ Han, L.-B.; Tanaka, M. J. Am. Chem. Soc. 1996, 118, 1571-1572.

${ }^{13}$ (a) Douglass, M. R.; Marks, T. J. J. Am. Chem. Soc. 2000, 122, 1824-1825. (b) Douglass, M. R.; Stern, C. L.; Marks, T. J. J. Am. Chem. Soc. 2001, 123, 10221-10238

${ }^{14}$ For an asymmetric version with activated alkenes, see: (a) Costa, E.; Pringle, P. G.; Smith, M. B.; Worboys, K. J. Chem. Soc., Dalton Trans. 1997, 4277-4282. (b) Costa, E.; Pringle, P. G.; Worboys, K. Chem. Commun. 1998, 49-50. (c) Kovacik, I.; Wicht, D. K.; Grewal, N. S.; Glueck, D. S.; Incarvito, C. D.; Guzei, I. A.; Rheingold, A. L. Organometallics 2000, 19, 950-953.

${ }^{15}$ For examples with alkynes, see: Kazankova, M. A.; Efimova, I. V.; Kochetkov, A. N.; Afanas'ev, V. V.; Beletskaya, I. P.; Dixneuf, P. H. Synlett 2001, 4, 497-500.
} 


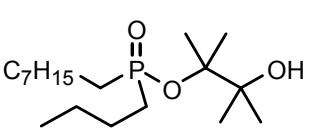

Butyl-octyl-phosphinic acid 2-hydroxy-1,1,2-trimethyl-propyl ester (5). To a cooled $\left(-78{ }^{\circ} \mathrm{C}\right)$ solution of $4(242 \mathrm{mg}, 1.0 \mathrm{mmol})$ in THF $(2.0 \mathrm{~mL})$ was added $2.27 \mathrm{M}$ BuLi in hexanes $(0.50 \mathrm{~mL}, 1.1 \mathrm{mmol})$. After stirring at $-78^{\circ} \mathrm{C}$ for $1 \mathrm{~h}$ the reaction mixture was concentrated in vacuo. Flash chromatography $\left(\mathrm{SiO}_{2}\right.$, gradient: EtOAc) afforded $5(31 \mathrm{mg}, 0.093 \mathrm{mmol})$ as a light yellow oil in $9 \%$ yield. $\mathrm{R}_{f} 0.40$ (EtOAc); ${ }^{1} \mathrm{H}$ NMR $\left(\mathrm{CDCl}_{3}, 300 \mathrm{MHz}\right) \delta$ 0.86-0.96 (m, 6H), 1.24-1.56 (m, 30H), 1.71-1.74 (m, 2H), 4.06 (brs, $\left.1 \mathrm{H}\right)$; ${ }^{13} \mathrm{C}$ NMR $\left(\mathrm{CDCl}_{3}, 75.5 \mathrm{MHz}\right) \delta 13.18\left(\mathrm{CH}_{3}\right), 14.3\left(\mathrm{CH}_{3}\right), 22.7\left(\mathrm{CH}_{2}\right), 22.9\left(\mathrm{CH}_{2}\right), 24.2\left(\mathrm{CH}_{2}\right), 24.8\left(\mathrm{CH}_{2}\right)$, $25.2\left(\mathrm{CH}_{3}\right), 25.5\left(\mathrm{CH}_{3}\right), 29.3\left(\mathrm{CH}_{2}\right), 31.3\left(\mathrm{CH}_{2}\right), 32.0\left(\mathrm{CH}_{2}\right), 74.6(\mathrm{C}), 91.3(\mathrm{C}), 91.4(\mathrm{C}) ;{ }^{31} \mathrm{P} \mathrm{NMR}\left(\mathrm{CDCl}_{3}\right.$, $121.5 \mathrm{MHz}) \delta 58.5$; MS (EI) $\mathrm{m} / z 335[\mathrm{M}+\mathrm{H}]^{+}$, $235[\mathrm{M}-t \mathrm{BuC}(\mathrm{O}) \mathrm{Me}+\mathrm{H}]^{+}$; MS (EI) $\mathrm{m} / z 371$ and 369 $[\mathrm{M}+\mathrm{Cl}]^{-}$; HRMS (CI) calcd for $\mathrm{C}_{18} \mathrm{H}_{39} \mathrm{O}_{3} \mathrm{P}[\mathrm{M}+\mathrm{H}]^{+} 335.2715$, found 335.2725 .

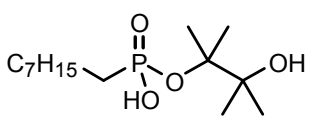

Octyl-Phosphonic acid mono-(2-hydroxy-1,1,2-trimethyl-propyl) ester (6). To a cooled $\left.-78^{\circ} \mathrm{C}\right)$ solution of $4(61 \mathrm{mg}, 0.25 \mathrm{mmol})$ in THF $(1.0 \mathrm{~mL})$ was added KHMDS in THF $(0.94 \mathrm{M}, 0.29 \mathrm{~mL}, 0.25 \mathrm{mmol})$. After stirring at $-78^{\circ} \mathrm{C}$ for $1 \mathrm{~h}, \mathrm{AcOH}(0.018 \mathrm{~mL}, 0.31 \mathrm{mmol})$ was added and the mixtrure was stirred for $16 \mathrm{~h}$. The resulting suspension was concentrated in vacuo, redissolved in $50 \% \mathrm{MeOH}$ in $\mathrm{CHCl}_{3}$ and filtered through silica gel. Concentrated in vacuo afforded crude 6. $\mathrm{R}_{f} 0.32\left(50 \% \mathrm{MeOH}\right.$ in $\left.\mathrm{CHCl}_{3}\right) ;{ }^{13} \mathrm{C}$ NMR $\left(\mathrm{CDCl}_{3}, 75.5 \mathrm{MHz}\right) \delta 14.3\left(\mathrm{CH}_{3}\right), 22.9\left(\mathrm{CH}_{2}\right), 24.0\left(\mathrm{CH}_{2}\right), 29.0\left(\mathrm{CH}_{2}\right), 30.9\left(\mathrm{CH}_{2}\right), 31.3\left(\mathrm{CH}_{2}\right), 31.6$ $\left(\mathrm{CH}_{2}\right), 32.1\left(\mathrm{CH}_{2}\right), 22.3\left(\mathrm{CH}_{3}\right), 24.5\left(\mathrm{CH}_{3}\right), 25.2\left(\mathrm{CH}_{3}\right), 75.8(\mathrm{C}), 85.8(\mathrm{C}), 85.9(\mathrm{C})$; MS (EI) $\mathrm{m} / z 295$ $[\mathrm{M}+\mathrm{H}]^{+}, 277\left[\mathrm{M}-\mathrm{H}_{2} \mathrm{O}+\mathrm{H}\right]^{+}, 195[\mathrm{M}-t \mathrm{BuC}(\mathrm{O}) \mathrm{Me}+\mathrm{H}]^{+}$; MS (EI) $\mathrm{m} / z 293[\mathrm{M}-\mathrm{H}]^{-}$; HRMS (CI) calcd for $\mathrm{C}_{14} \mathrm{H}_{31} \mathrm{O}_{4} \mathrm{P}[\mathrm{M}+\mathrm{H}]^{+}$295.2038, found 295.2050.

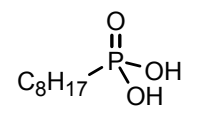

Octyl-Phosphonic acid (7). To a solution of $4(187 \mathrm{mg}, 0.68 \mathrm{mmol})$ in $\mathrm{CH}_{2} \mathrm{Cl}_{2}(2.0 \mathrm{~mL})$ was added TMSBr $(0.35 \mathrm{~mL}, 2.65 \mathrm{mmol})$ and the mixture was stirred at $\mathrm{rt}$ for $16 \mathrm{~h}$ followed by concentration in vacuo. The residue was redissolved in $\mathrm{CH}_{2} \mathrm{Cl}_{2}(1.5 \mathrm{~mL})$ and $\mathrm{MeOH}(0.5 \mathrm{~mL})$ was then added. The clear solution was allowed to stir at $\mathrm{rt}$ for $2 \mathrm{~h}$ followed by concentration in vacuo to yield crude $7 .{ }^{1} \mathrm{H} \mathrm{NMR}\left(\mathrm{CDCl}_{3}, 300 \mathrm{MHz}\right) \delta 0.87(\mathrm{t}, \mathrm{J}=6.6 \mathrm{~Hz}, 3 \mathrm{H})$, 1.21-2.01 (m, 12), 9.65 (brs, $2 \mathrm{H}) ;{ }^{13} \mathrm{C} \mathrm{NMR}\left(\mathrm{CDCl}_{3}, 75.5 \mathrm{MHz}\right) \delta 14.3\left(\mathrm{CH}_{3}\right), 22.1\left(\mathrm{CH}_{2}\right), 22.8\left(\mathrm{CH}_{2}\right), 24.9$ $\left(\mathrm{CH}_{2}\right), 25.0\left(\mathrm{CH}_{2}\right), 26.8\left(\mathrm{CH}_{2}\right), 29.2\left(\mathrm{CH}_{2}\right), 29.3\left(\mathrm{CH}_{2}\right), 29.9\left(\mathrm{CH}_{2}\right), 30.5\left(\mathrm{CH}_{2}\right), 30.8\left(\mathrm{CH}_{2}\right), 32.0\left(\mathrm{CH}_{2}\right) ;{ }^{31} \mathrm{P}$ NMR $\left(\mathrm{CDCl}_{3}, 121.5 \mathrm{MHz}\right) \delta 38.0$; MS (EI) $\mathrm{m} / z 195[\mathrm{M}+\mathrm{H}]^{+}$.
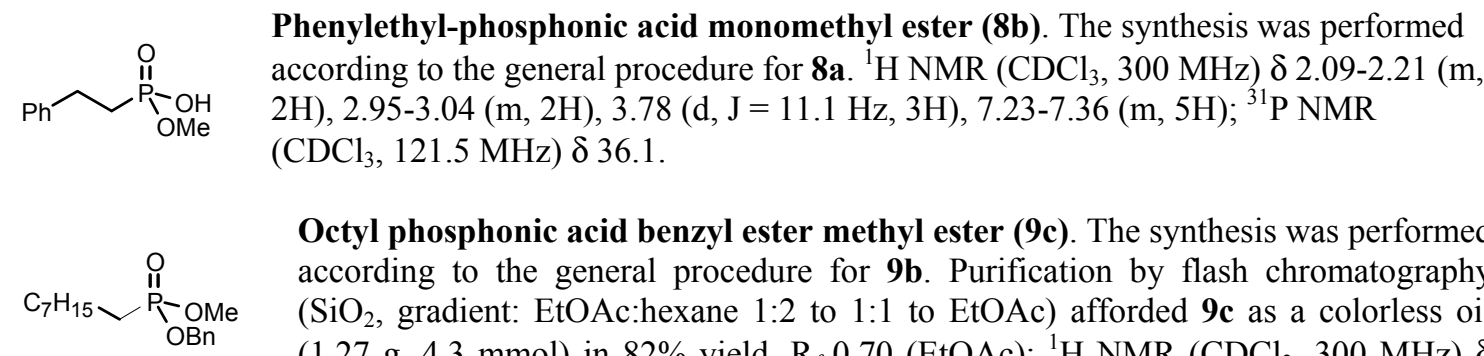

Octyl phosphonic acid benzyl ester methyl ester (9c). The synthesis was performed according to the general procedure for $\mathbf{9 b}$. Purification by flash chromatography ( $\mathrm{SiO}_{2}$, gradient: EtOAc:hexane 1:2 to $1: 1$ to EtOAc) afforded $9 \mathbf{c}$ as a colorless oil $(1.27 \mathrm{~g}, 4.3 \mathrm{mmol})$ in $82 \%$ yield. $\mathrm{R}_{f} 0.70$ (EtOAc); ${ }^{1} \mathrm{H} \mathrm{NMR}\left(\mathrm{CDCl}_{3}, 300 \mathrm{MHz}\right) \delta$ $0.89(\mathrm{t}, \mathrm{J}=6.8 \mathrm{~Hz}, 3 \mathrm{H}), 1.26-1.36(\mathrm{~m}, 10 \mathrm{H}), 1.54-1.80(\mathrm{~m}, 4 \mathrm{H}), 3.68(\mathrm{~d}, \mathrm{~J}=10.8 \mathrm{~Hz}, 3 \mathrm{H}), 5.09(\mathrm{~d}, \mathrm{~J}=8.4$ $\mathrm{Hz}, 2 \mathrm{H}), 7.28-7.42(\mathrm{~m}, 5 \mathrm{H}) ;{ }^{13} \mathrm{C} \mathrm{NMR}\left(\mathrm{CDCl}_{3}, 75.5 \mathrm{MHz}\right) \delta 14.3\left(\mathrm{CH}_{3}\right), 22.5\left(\mathrm{CH}_{2}\right), 22.6\left(\mathrm{CH}_{2}\right), 22.9$ $\left(\mathrm{CH}_{2}\right)$, $24.7\left(\mathrm{CH}_{2}\right), 26.6\left(\mathrm{CH}_{2}\right), 29.2\left(\mathrm{CH}_{2}\right), 30.7\left(\mathrm{CH}_{2}\right), 30.9\left(\mathrm{CH}_{2}\right), 32.1\left(\mathrm{CH}_{2}\right), 52.3\left(\mathrm{CH}_{3}\right), 67.3\left(\mathrm{CH}_{2}\right)$, $67.4\left(\mathrm{CH}_{2}\right), 128.1(\mathrm{CH}), 128.6(\mathrm{CH}), 128.8(\mathrm{CH}), 136.8(\mathrm{C}), 136.9(\mathrm{C}) ;{ }^{31} \mathrm{P}$ NMR $\left(\mathrm{CDCl}_{3}, 121.5 \mathrm{MHz}\right) \delta$ 35.7; MS (CI) $m / z 299[\mathrm{M}+\mathrm{H}]^{+}$; HRMS (CI) calcd for $\mathrm{C}_{16} \mathrm{H}_{27} \mathrm{O}_{3} \mathrm{P}[\mathrm{M}+\mathrm{H}]^{+}$299.1776, found 299.1773.

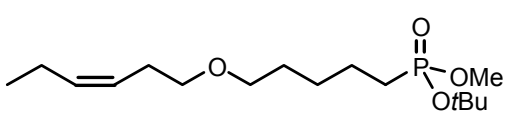

(5-Hex-3-enyloxy-pentyl)-phosphonic acid tert-butyl ester methyl ester (9e). The synthesis was performed according to the general procedure for 9d. Purification by flash chromatography $\left(\mathrm{SiO}_{2}\right.$, gradient: EtOAc:hexane 1:2 to 1:1 to EtOAc) afforded 9e $(1.21 \mathrm{~g}, 3.8 \mathrm{mmol})$ as a colorless oil in $88 \%$ yield. $\mathrm{R}_{f} 0.52(\mathrm{EtOAc}) ;{ }^{1} \mathrm{H} \mathrm{NMR}\left(\mathrm{CDCl}_{3}, 300 \mathrm{MHz}\right) \delta 0.89(\mathrm{t}$, $\mathrm{J}=7.4 \mathrm{~Hz}, 3 \mathrm{H}), 1.33-1.67(\mathrm{~m}, 8 \mathrm{H}), 1.43(\mathrm{~s}, 9 \mathrm{H}), 1.92-2.04(\mathrm{~m}, 2 \mathrm{H}), 2.15-2.28(\mathrm{~m}, 2 \mathrm{H}), 3.31-3.36(\mathrm{~m}$, $4 \mathrm{H}), 3.62(\mathrm{~d}, \mathrm{~J}=11.1 \mathrm{~Hz}, 3 \mathrm{H}), 5.22-5.49(\mathrm{~m}, 2 \mathrm{H}) ;{ }^{31} \mathrm{P} \mathrm{NMR}\left(\mathrm{CDCl}_{3}, 121.5 \mathrm{MHz}\right) \delta 30.6$. 


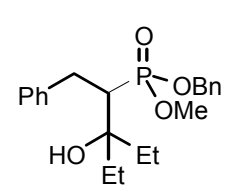

(1-Benzyl-2-ethyl-2-hydroxy-butyl)-phosphonic acid benzyl ester methyl ester (10b). The synthesis was performed according to the general procedure for 10a. Purification by flash chromatography $\left(\mathrm{SiO}_{2}\right.$, gradient: EtOAc:hexane $1: 4$ to $\left.1: 1\right)$ afforded $10 \mathrm{~b}(380 \mathrm{mg}, 1.01 \mathrm{mmol})$ as a colorless oil in $62 \%$ yield. $\mathrm{R}_{f} 0.70$ (EtOAc);

${ }^{1} \mathrm{H}$ NMR $\left(\mathrm{CDCl}_{3}, 300 \mathrm{MHz}\right) \delta$ 0.92-1.03 (m, 6H), 1.52-1.90 (m. 4H), 2.45-2.65 (m, $1 \mathrm{H}), 2.80-2.95(\mathrm{~m}, 2 \mathrm{H}), 3.28$ and $3.64(\mathrm{~d}, \mathrm{~J}=10.8 \mathrm{~Hz}, 3 \mathrm{H}), 4.46-5.05(\mathrm{~m}, 3 \mathrm{H}), 7.03-7.40(\mathrm{~m}, 10 \mathrm{H}) ;{ }^{13} \mathrm{C}$ NMR $\left(\mathrm{CDCl}_{3}, 75.5 \mathrm{MHz}\right) \delta 6.94\left(\mathrm{CH}_{3}\right), 6.98\left(\mathrm{CH}_{3}\right), 7.25\left(\mathrm{CH}_{3}\right), 7.32\left(\mathrm{CH}_{3}\right), 27.97\left(\mathrm{CH}_{2}\right), 28.07\left(\mathrm{CH}_{2}\right)$, $28.13\left(\mathrm{CH}_{2}\right), 28.24\left(\mathrm{CH}_{2}\right), 30.22\left(\mathrm{CH}_{2}\right), 30.28\left(\mathrm{CH}_{2}\right), 30.31\left(\mathrm{CH}_{2}\right), 30.37\left(\mathrm{CH}_{2}\right), 31.79\left(\mathrm{CH}_{2}\right), 31.85\left(\mathrm{CH}_{2}\right)$, $31.93\left(\mathrm{CH}_{2}\right), 31.99\left(\mathrm{CH}_{2}\right), 45.1(\mathrm{CH}), 46.2(\mathrm{CH}), 46.9(\mathrm{CH}), 47.9(\mathrm{CH}), 51.45\left(\mathrm{CH}_{3}\right), 51.55\left(\mathrm{CH}_{3}\right), 51.48$ $\left(\mathrm{CH}_{3}\right), 51.66\left(\mathrm{CH}_{3}\right), 66.72\left(\mathrm{CH}_{2}\right), 66.81\left(\mathrm{CH}_{2}\right), 66.96\left(\mathrm{CH}_{2}\right), 67.04\left(\mathrm{CH}_{2}\right), 74.95\left(\mathrm{CH}_{2}\right), 74.99\left(\mathrm{CH}_{2}\right)$, $125.93(\mathrm{CH}), 125.95(\mathrm{CH}), 126.53(\mathrm{CH}), 126.86(\mathrm{CH}), 127.80(\mathrm{CH}), 127.90(\mathrm{CH}), 127.97(\mathrm{CH}), 128.00$ $(\mathrm{CH}), 128.10(\mathrm{CH}), 128.20(\mathrm{CH}), 128.32(\mathrm{CH}), 128.50(\mathrm{CH}), 128.63(\mathrm{CH}), 135.38(\mathrm{C}), 135.45(\mathrm{C}), 135.74$ (C), 135.82 (C), 139.51 (C), 139.55 (C), 139.61 (C); ${ }^{31} \mathrm{P}$ NMR $\left(\mathrm{CDCl}_{3}, 121.5 \mathrm{MHz}\right) \delta 36.6,36.5 ; \mathrm{MS}$ (CI) $\mathrm{m} / z 377[\mathrm{M}+\mathrm{H}]^{+}, 359\left[\mathrm{M}-\mathrm{H}_{2} \mathrm{O}+\mathrm{H}\right]^{+}$; HRMS (CI) calcd for $\mathrm{C}_{21} \mathrm{H}_{30} \mathrm{O}_{4} \mathrm{P}[\mathrm{M}+\mathrm{H}]^{+}$377.1882, found 377.1881.

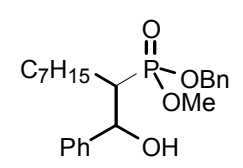

[1-(Hydroxy-phenyl-methyl)-octyl]-phosphonic acid benzyl ester methyl ester (10c). The synthesis was performed according to the general procedure for 10a. Purification by flash chromatography $\left(\mathrm{SiO}_{2}\right.$, gradient: EtOAc:hexane 1:2 to 1:1) afforded $10 \mathrm{c}$ ( $322 \mathrm{mg}, 0.80 \mathrm{mmol}$ ) as a colorless oil in $80 \%$ yield. $\mathrm{R}_{f} 0.35$ (EtOAc:hexane 1:1); ${ }^{1} \mathrm{H}$ NMR $\left(\mathrm{CDCl}_{3}, 300 \mathrm{MHz}\right) \delta 0.84(\mathrm{t}, \mathrm{J}=6.8 \mathrm{~Hz} .3 \mathrm{H}), 1.06-$ $1.70(\mathrm{~m}, 12 \mathrm{H}), 2.09-2.19(\mathrm{~m}, 1 \mathrm{H}), 3.56-3.64(\mathrm{~m}, 3 \mathrm{H}), 4.07-5.38(\mathrm{~m}, 3 \mathrm{H}), 7.19-7.40(\mathrm{~m}, 5 \mathrm{H}) ;{ }^{31} \mathrm{P}$ NMR $\left(\mathrm{CDCl}_{3}, 121.5 \mathrm{MHz}\right) \delta 35.9,36.1,36.4$; MS (CI) $\mathrm{m} / z$ $405[\mathrm{M}+\mathrm{H}]^{+}, 387\left[\mathrm{M}-\mathrm{H}_{2} \mathrm{O}+\mathrm{H}\right]^{+}$; HRMS (CI) calcd for $\mathrm{C}_{23} \mathrm{H}_{33} \mathrm{O}_{4} \mathrm{P}[\mathrm{M}+\mathrm{H}]^{+}$405.2195, found 405.2197.

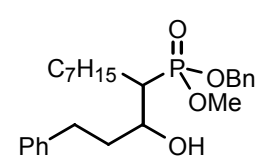

[1-(1-Hydroxy-3-phenyl-propyl)-octyl]-phosphonic acid benzyl ester methyl ester (10d). The synthesis was performed according to the general procedure for 10a. Purification by flash chromatography $\left(\mathrm{SiO}_{2}\right.$, gradient: EtOAc:hexane 1:2 to $1: 1$ to EtOAc) afforded $10 d(247 \mathrm{mg}, 0.57 \mathrm{mmol})$ as a colorless oil in $57 \%$ yield. $\mathrm{R}_{f}$ 0.74 (EtOAc); ${ }^{1} \mathrm{H}$ NMR $\left(\mathrm{CDCl}_{3}, 300 \mathrm{MHz}\right) \delta 0.91(\mathrm{t}, \mathrm{J}=6.8 \mathrm{~Hz}, 3 \mathrm{H}), 1.25-2.06(\mathrm{~m}$, $15 \mathrm{H}), 2.60-2.97(\mathrm{~m}, 2 \mathrm{H}), 3.63-3.73(\mathrm{~m}, 3 \mathrm{H}), 3.83-3.99(\mathrm{~m}, 1 \mathrm{H}), 5.07-5.13(\mathrm{~m}, 2 \mathrm{H}), 7.17-7.40(\mathrm{~m}, 5 \mathrm{H}) ;{ }^{31} \mathrm{P}$ NMR $\left(\mathrm{CDCl}_{3}, 121.5 \mathrm{MHz}\right) \delta 36.0,36.2,36.4,36.4$; MS (CI) $\mathrm{m} / z 433[\mathrm{M}+\mathrm{H}]^{+}, 415\left[\mathrm{M}-\mathrm{H}_{2} \mathrm{O}+\mathrm{H}\right]^{+}$; HRMS (CI) calcd for $\mathrm{C}_{25} \mathrm{H}_{37} \mathrm{O}_{4} \mathrm{P}[\mathrm{M}+\mathrm{H}]^{+} 433.2508$, found 433.2500 .

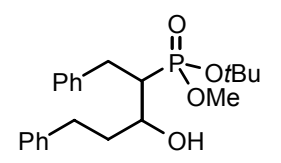

(1-Benzyl-2-hydroxy-4-phenyl-butyl)- phosphonic acid tert-butyl ester methyl ester (10e). The synthesis was performed according to the general procedure for 10a. Purification by flash chromatography $\left(\mathrm{SiO}_{2}\right.$, gradient: EtOAc:hexane 1:4 to 1:2) afforded $10 \mathrm{e}(206 \mathrm{mg}, 0.53 \mathrm{mmol})$ as a colorless oil in $53 \%$ yield. $\mathrm{R}_{f} 0.65$ (EtOAc); ${ }^{31} \mathrm{P}$ NMR $\left(\mathrm{CDCl}_{3}, 121.5 \mathrm{MHz}\right) \delta 30.1,30.3,30.4$; MS (CI) $\mathrm{m} / z 391$ $[\mathrm{M}+\mathrm{H}]^{+}$; HRMS (CI) calcd for $\mathrm{C}_{22} \mathrm{H}_{31} \mathrm{O}_{4} \mathrm{P}[\mathrm{M}+\mathrm{H}]^{+}$391.2038, found 391.2026.

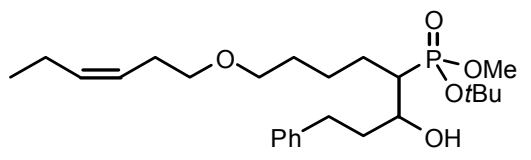

[5-Hex-3-enyloxy-1-(5-Hex-3-enyloxy-pentyl)]-phosphonic acid tert-butyl ester methyl ester (10f). The synthesis was performed according to the general procedure for $\mathbf{1 0 a}$. Purification by flash chromatography $\left(\mathrm{SiO}_{2}\right.$, gradient: EtOAc:hexane $1: 2$ to $1: 1)$ afforded $\mathbf{1 0 f}(338 \mathrm{mg}, 0.75 \mathrm{mmol})$ as a colorless oil in $82 \%$ yield. $\mathrm{R}_{f} 0.56(\mathrm{EtOAc}) ;{ }^{1} \mathrm{H} \mathrm{NMR}\left(\mathrm{CDCl}_{3}, 300 \mathrm{MHz}\right) \delta 0.95(\mathrm{t}, \mathrm{J}=7.5 \mathrm{~Hz}, 3 \mathrm{H}), 1.14-$ $2.08(\mathrm{~m}, 12 \mathrm{H}), 1.47(\mathrm{~s}, 9 \mathrm{H}), 2.19-2.32(\mathrm{~m}, 2 \mathrm{H}), 2.58-2.72(\mathrm{~m}, 1 \mathrm{H}), 2.83-2.91(\mathrm{~m}, 1 \mathrm{H}), 3.35-3.39(\mathrm{~m}, 4 \mathrm{H})$, 3.64-4.10 (m, 5H), 5.27-5.53 (m, 2H), 7.12-7.28 (m,5H); ${ }^{31} \mathrm{P} \mathrm{NMR}\left(\mathrm{CDCl}_{3}, 121.5 \mathrm{MHz}\right) \delta 31.4,31.6$; MS (CI) $m / z 455[\mathrm{M}+\mathrm{H}]^{+}$; HRMS (CI) calcd for $\mathrm{C}_{25} \mathrm{H}_{43} \mathrm{O}_{5} \mathrm{P}[\mathrm{M}+\mathrm{H}]^{+}$455.2926, found 455.2920.

1-Phenyl-1-nonene (12c). The synthesis was performed according to the general $\mathrm{C}_{7} \mathrm{H}_{15} \sim^{\mathrm{Ph}}$ procedure for 12b. Purification by flash chromatography $\left(\mathrm{SiO}_{2}\right.$, hexane) afforded 12c (99 $\mathrm{mg}, 0.38 \mathrm{mmol}$ ) as a colorless oil in $59 \%$ yield. $\mathrm{R}_{f} 0.65$ (hexanes); ${ }^{1} \mathrm{H} \mathrm{NMR}$ $\left(\mathrm{CDCl}_{3}, 300 \mathrm{MHz}\right) \delta$ 0.94-0.98 (m. 3H), 1.35-1.55 (m, 10H), 2.25-2.45 (m, 2H), 5.70-5.79 (m, 0.8H), 6.25- 
$6.35(\mathrm{~m}, 0.2 \mathrm{H}), 6.43-6.51(\mathrm{~m}, 1 \mathrm{H}), 7.23-7.43(\mathrm{~m}, 5 \mathrm{H}) ;{ }^{13} \mathrm{C} \mathrm{NMR}\left(\mathrm{CDCl}_{3}, 75.5 \mathrm{MHz}\right) \delta 14.4\left(\mathrm{CH}_{3}\right), 23.0$ $\left(\mathrm{CH}_{2}\right), 29.0\left(\mathrm{CH}_{2}\right), 29.5\left(\mathrm{CH}_{2}\right), 29.6\left(\mathrm{CH}_{2}\right), 29.7\left(\mathrm{CH}_{2}\right), 30.3\left(\mathrm{CH}_{2}\right), 32.1\left(\mathrm{CH}_{2}\right), 33.4\left(\mathrm{CH}_{2}\right), 126.2(\mathrm{CH})$, $126.7(\mathrm{CH}), 127.0(\mathrm{CH}), 128.4(\mathrm{CH}), 128.7(\mathrm{CH}), 129.0(\mathrm{CH}), 130.0(\mathrm{CH}), 131.5(\mathrm{CH}), 133.6(\mathrm{CH}), 138.1$ (C); MS (CI) $m / z 203[\mathrm{M}+\mathrm{H}]^{+}$.

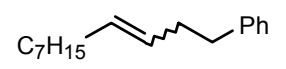

1-Phenyl-3-undecene (12d). The synthesis was performed according to the general procedure for $\mathbf{1 2 b}$. Purification by flash chromatography $\left(\mathrm{SiO}_{2}\right.$, hexane) afforded 12d (99 mg, $0.382 \mathrm{mmol}$ ) as a colorless oil in $55 \%$ yield. $\mathrm{R}_{f} 0.65$ (hexanes); ${ }^{1} \mathrm{H}$ NMR $\left(\mathrm{CDCl}_{3}, 300 \mathrm{MHz}\right) \delta$ 0.89-0.93 (m. 3H), 1.29 (brs 10H), 1.99 (brs, 2H), 2.35-2.42 (m, $2 \mathrm{H}), 2.66-2.72(\mathrm{~m}, 2 \mathrm{H}), 5.40-5.46(\mathrm{~m}, 2 \mathrm{H}), 7.18-7.33(\mathrm{~m}, 5 \mathrm{H}) ;{ }^{13} \mathrm{C} \mathrm{NMR}\left(\mathrm{CDCl}_{3}, 75.5 \mathrm{MHz}\right) \delta 14.4\left(\mathrm{CH}_{3}\right)$, $22.9\left(\mathrm{CH}_{2}\right), 27.5\left(\mathrm{CH}_{2}\right), 29.4\left(\mathrm{CH}_{2}\right), 29.5\left(\mathrm{CH}_{2}\right), 29.8\left(\mathrm{CH}_{2}\right), 29.9\left(\mathrm{CH}_{2}\right), 30.0\left(\mathrm{CH}_{2}\right), 32.1\left(\mathrm{CH}_{2}\right), 32.8$ $\left(\mathrm{CH}_{2}\right), 34.7\left(\mathrm{CH}_{2}\right), 36.3\left(\mathrm{CH}_{2}\right), 36.4\left(\mathrm{CH}_{2}\right), 125.9(\mathrm{CH}), 126.0(\mathrm{CH}), 128.5(\mathrm{CH}), 128.7(\mathrm{CH}), 128.9(\mathrm{CH})$, $129.5(\mathrm{CH}), 131.0(\mathrm{CH}), 131.5(\mathrm{CH}), 142.4(\mathrm{C})$; MS (CI) $\mathrm{m} / z 231[\mathrm{M}+\mathrm{H}]^{+}$.

(8-Hex-3-enyloxy-oct-3-enyl)-benzene (12f). The synthesis was

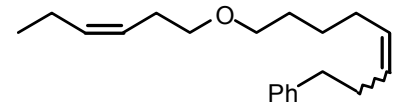
performed according to the general procedure for 12e. Purification by flash chromatography $\left(\mathrm{SiO}_{2}\right.$, gradient: hexane to EtOAc:hexane 1:19) afforded $\mathbf{1 2 f}(151 \mathrm{mg}, 0.53 \mathrm{mmol})$ as a colorless oil in $61 \%$ yield. $\mathrm{R}_{f} 0.33$ (EtOAc:hexane 1:19); ${ }^{1} \mathrm{H}$ NMR $\left(\mathrm{CDCl}_{3}, 300 \mathrm{MHz}\right) \delta 1.00-1.06(\mathrm{~m}, 3 \mathrm{H}), 1.34-1.49(\mathrm{~m}, 2 \mathrm{H}), 1.56-1.72(\mathrm{~m}$, $2 \mathrm{H}), \quad 2.04-2.16(\mathrm{~m}, 4 \mathrm{H}), 2.33-2.46(\mathrm{~m}, 4 \mathrm{H}), 2.70-2.76(\mathrm{~m}, 2 \mathrm{H}), 3.43-3.50(\mathrm{~m}, 4 \mathrm{H}), \quad 5.40-5.58(\mathrm{~m}, 4 \mathrm{H})$, 7.21-7.36 (m,5H); MS (CI) m/z $287[\mathrm{M}+\mathrm{H}]^{+}$; HRMS (CI) calcd for $\mathrm{C}_{20} \mathrm{H}_{30} \mathrm{O}[\mathrm{M}+\mathrm{H}]^{+}$287.2375, found 287.2369. 


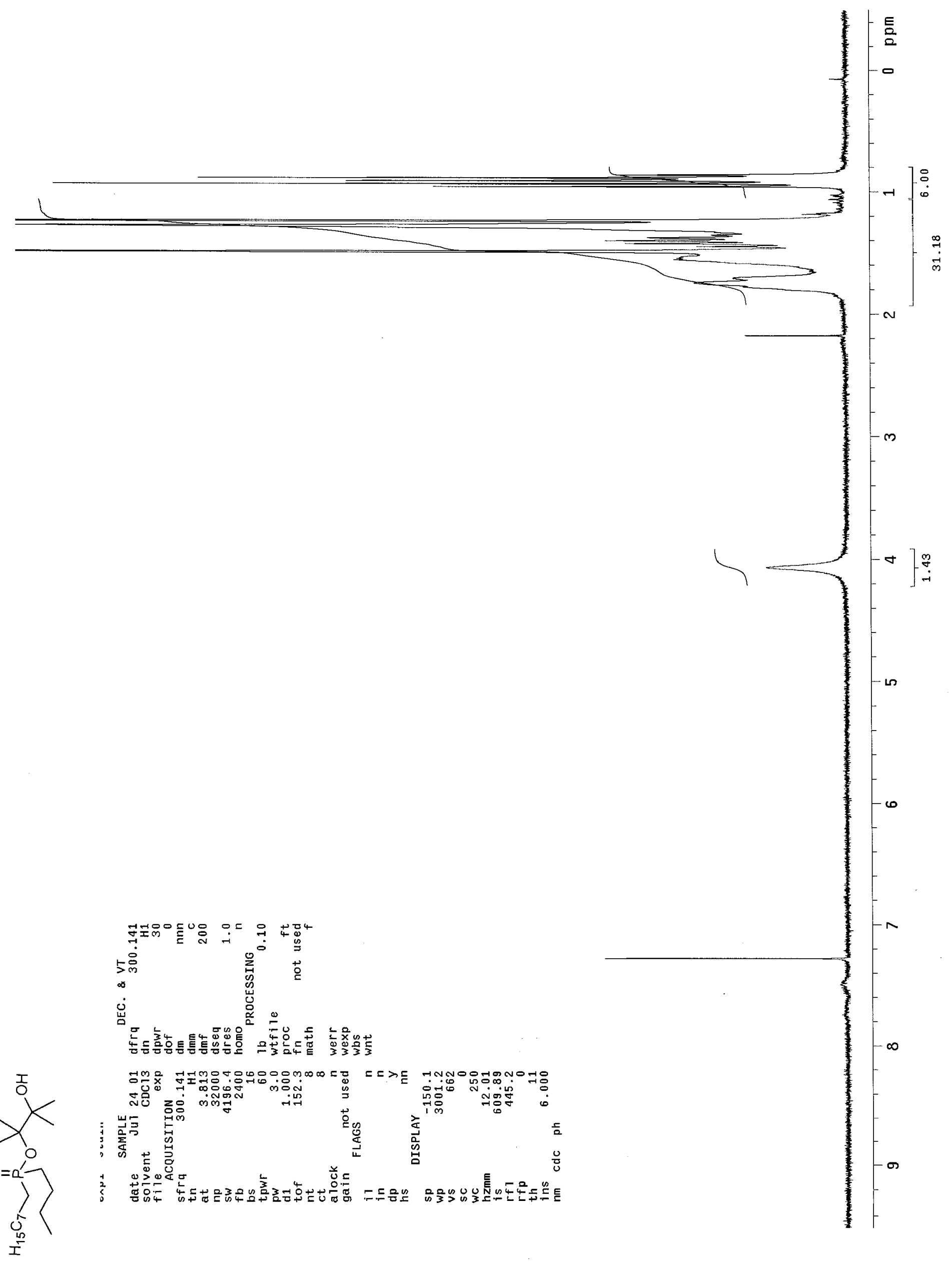




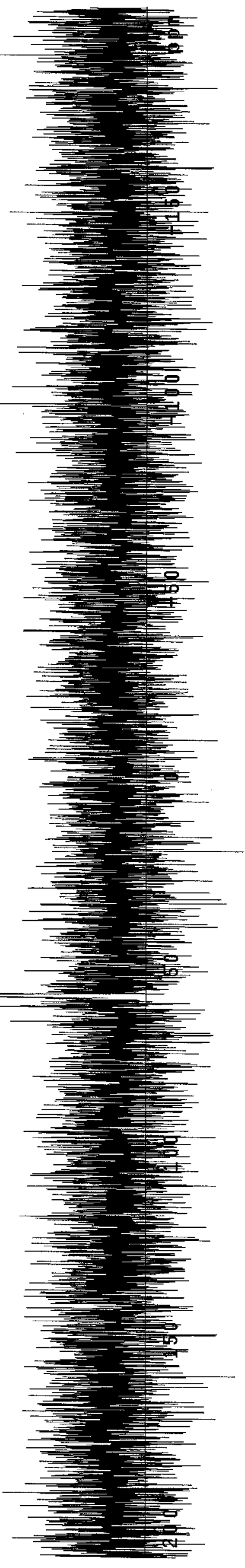




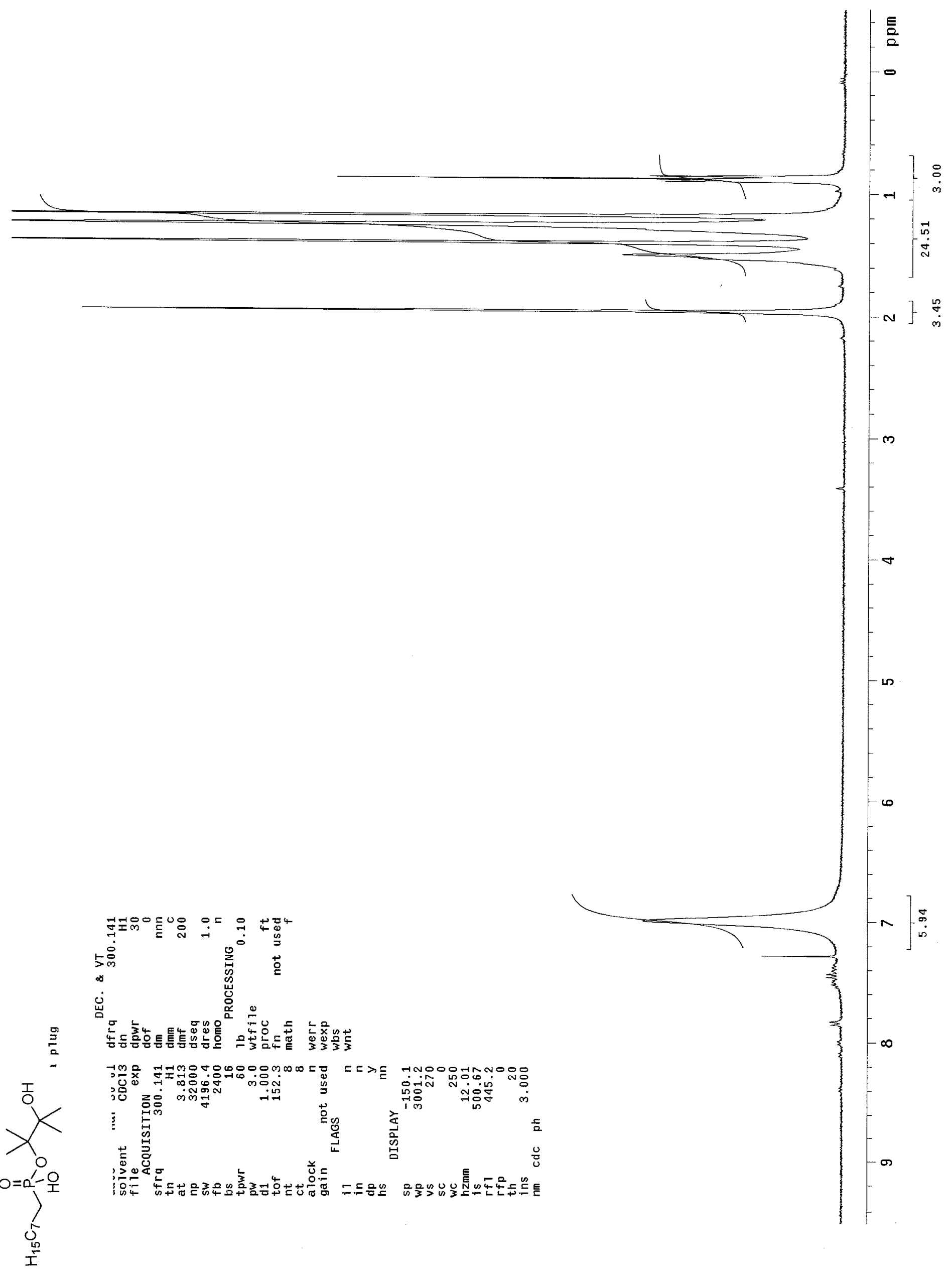




$$
1
$$




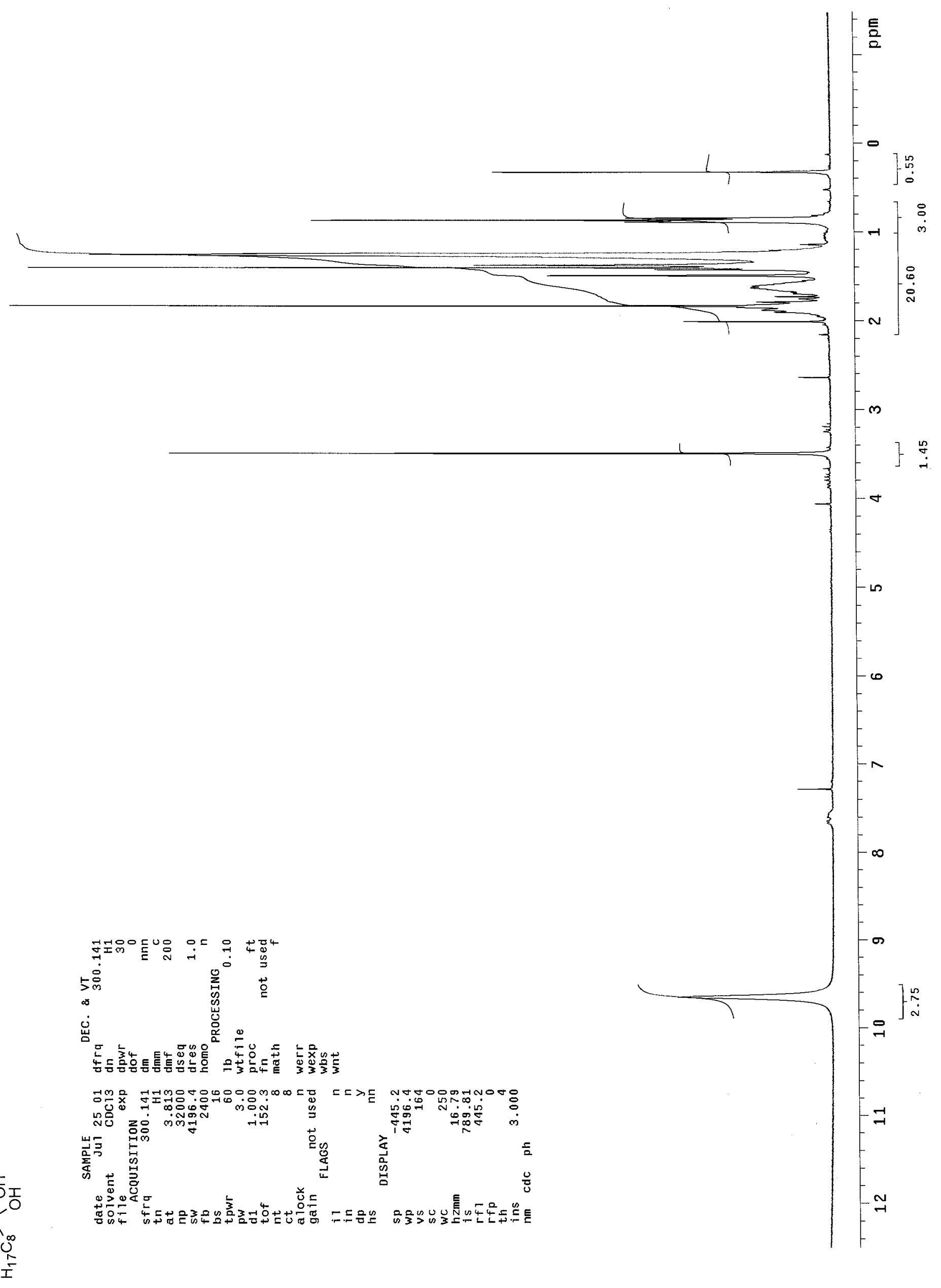




$$
1
$$




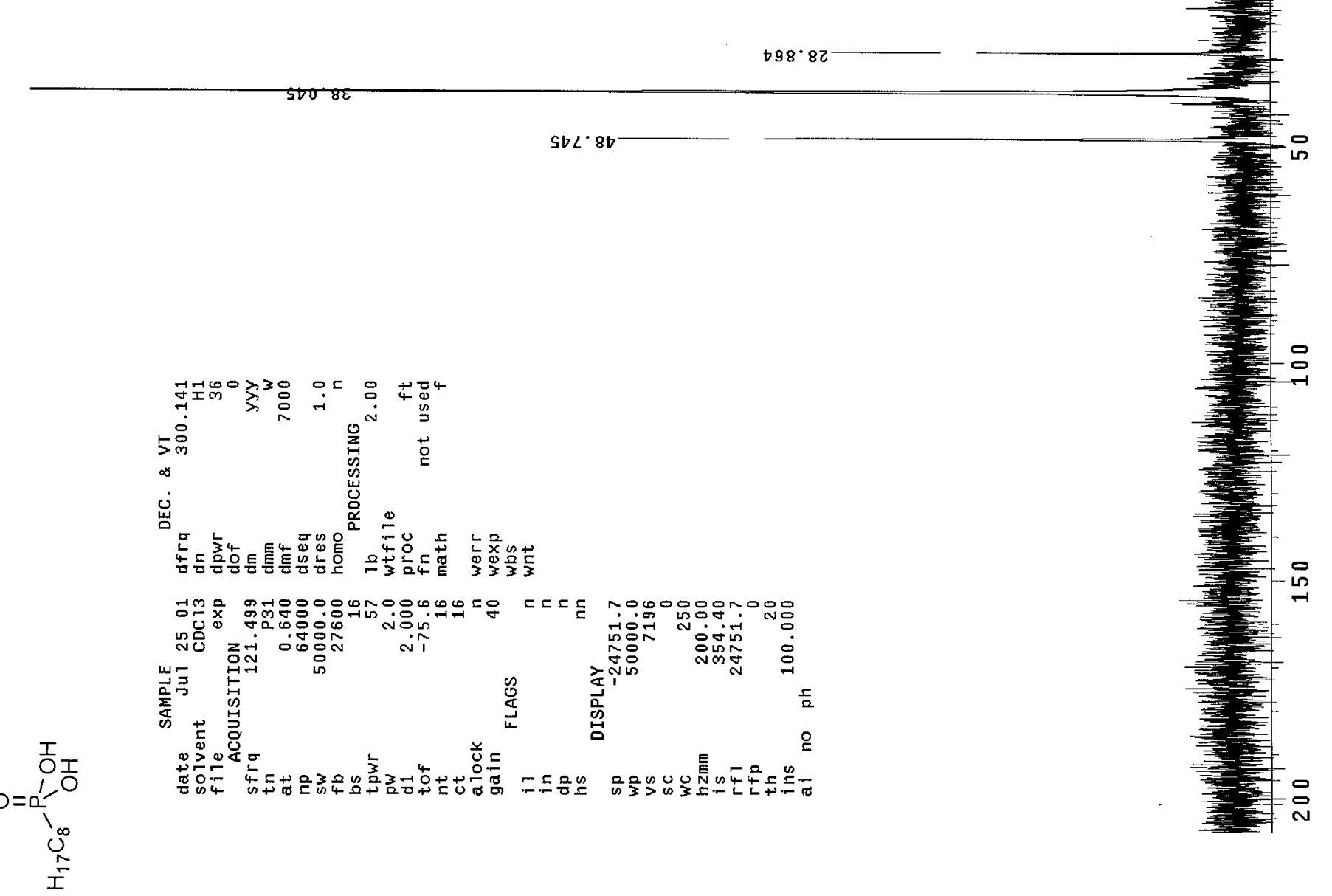




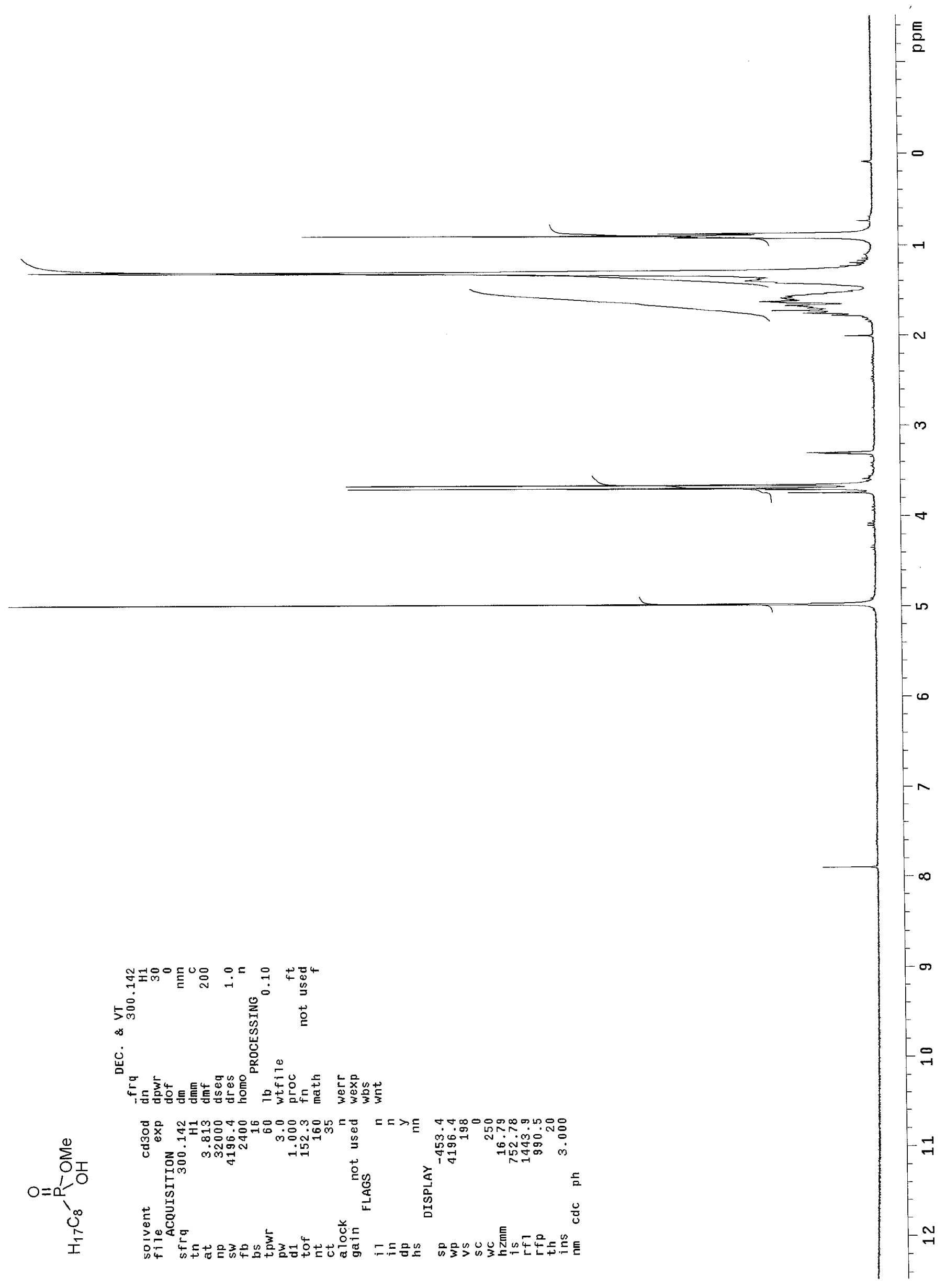




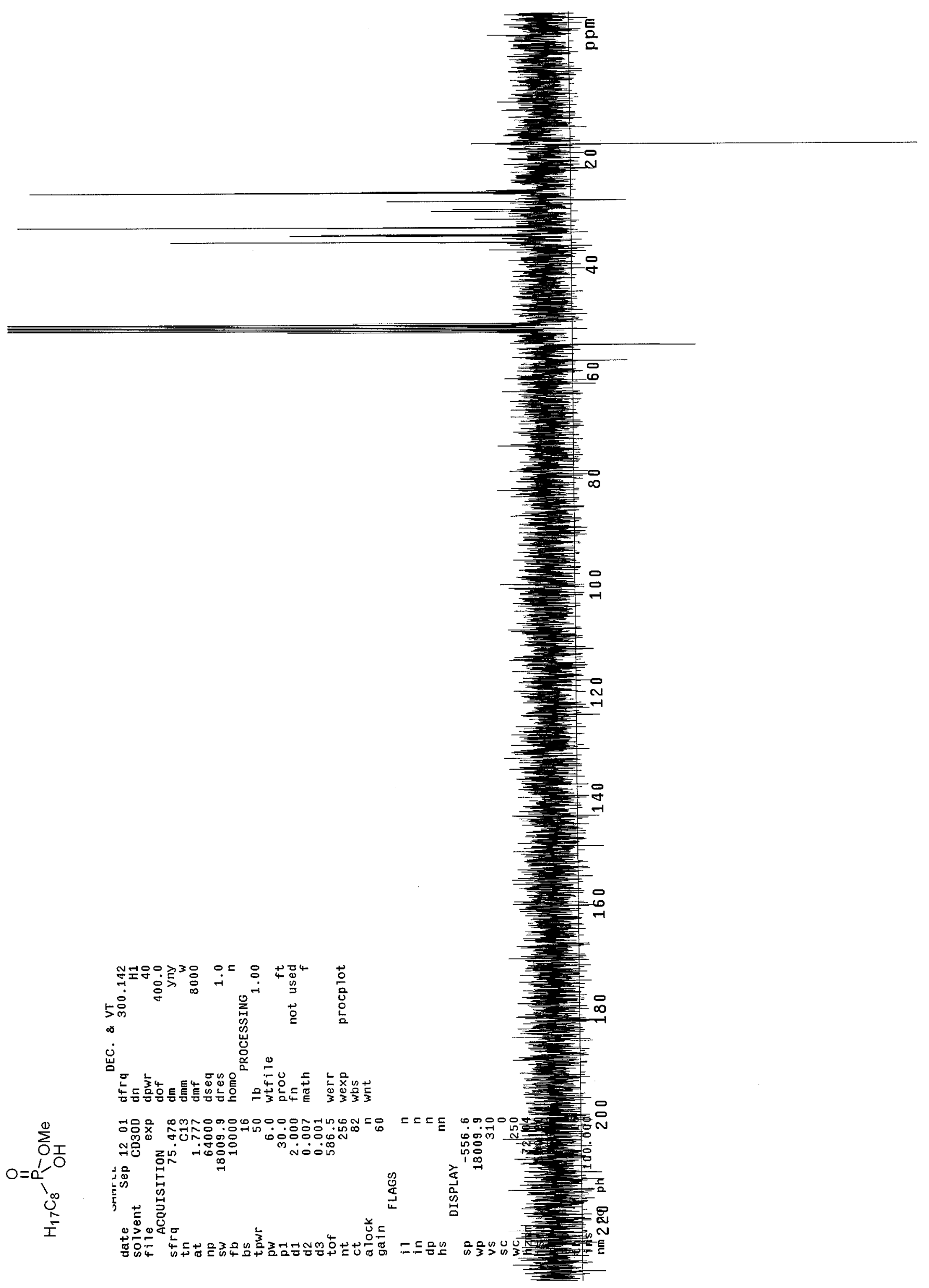




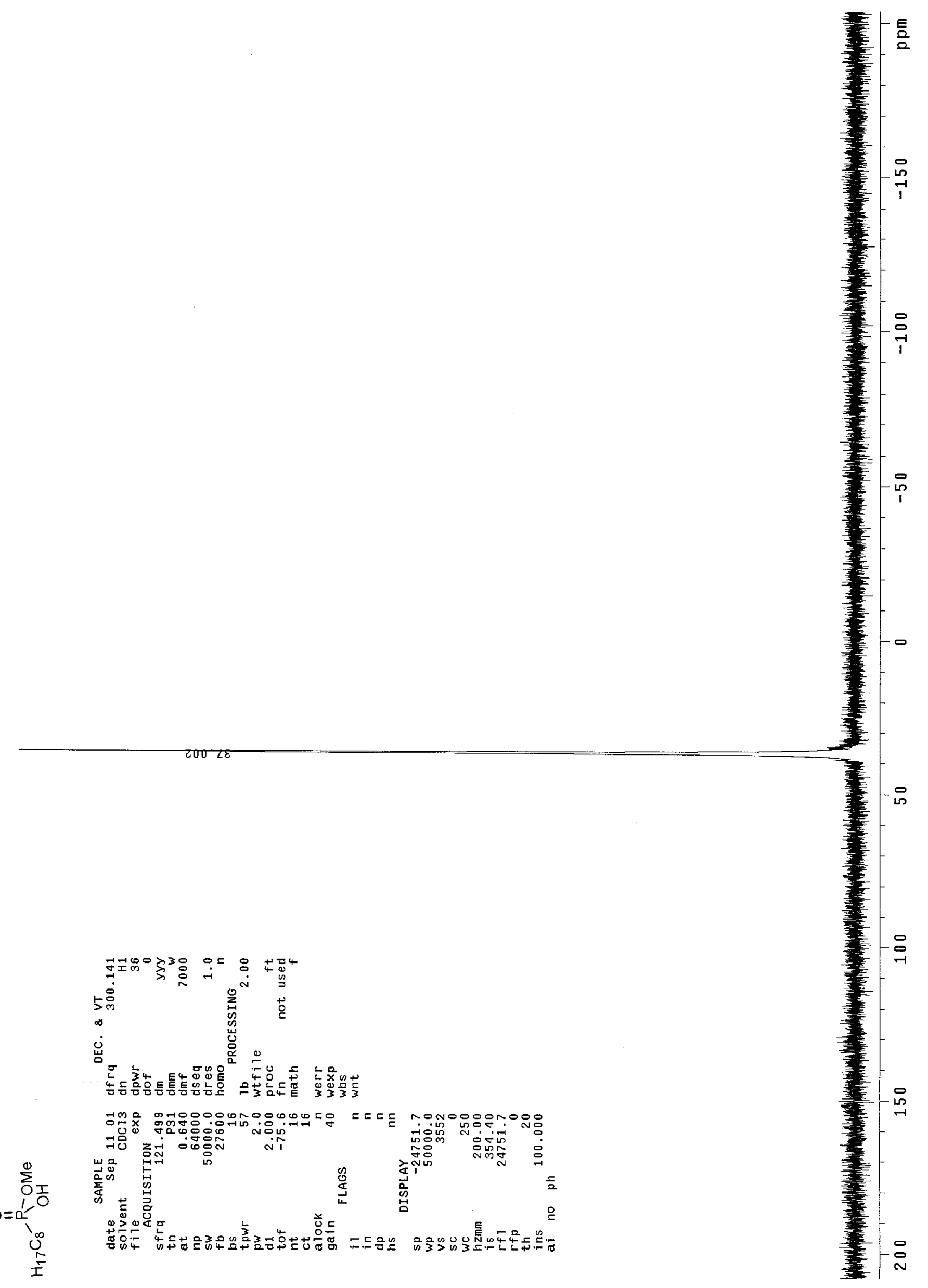




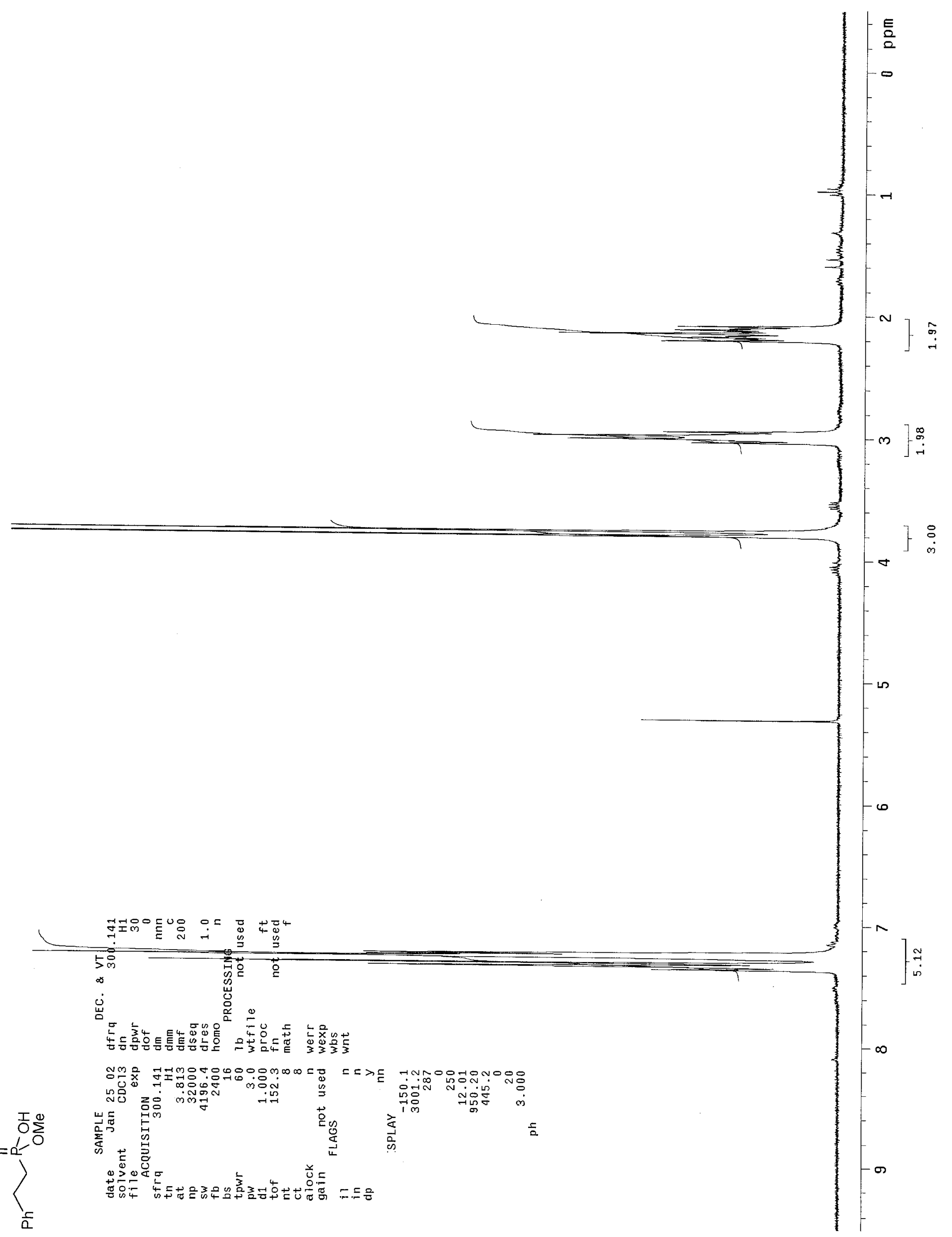




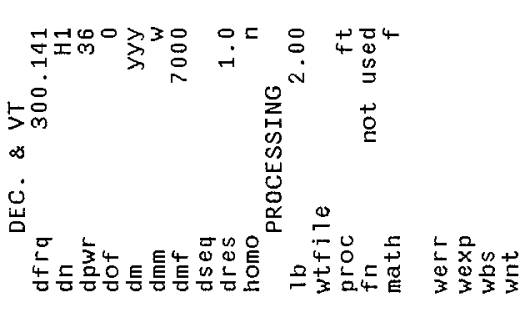

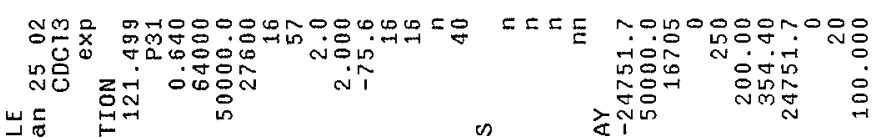
$0=0$

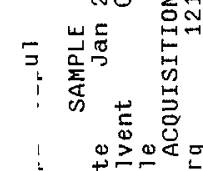

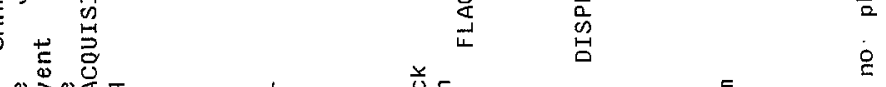

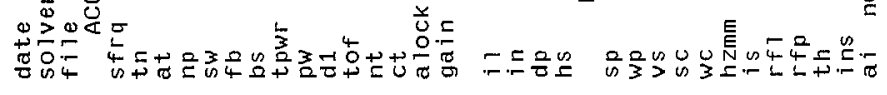

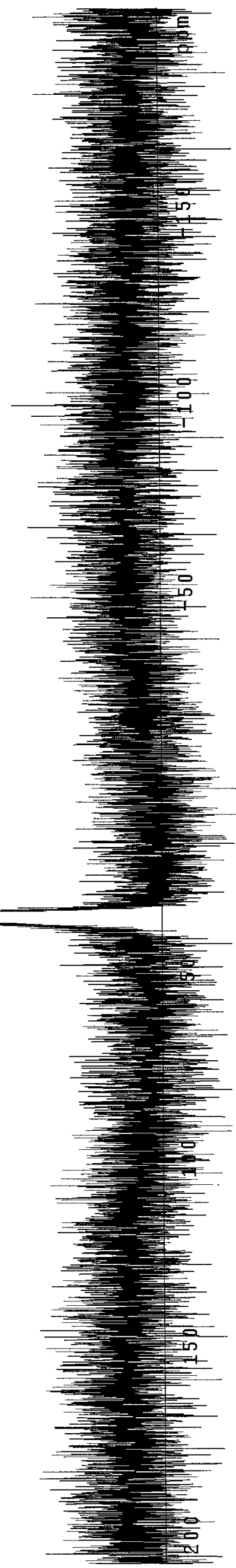




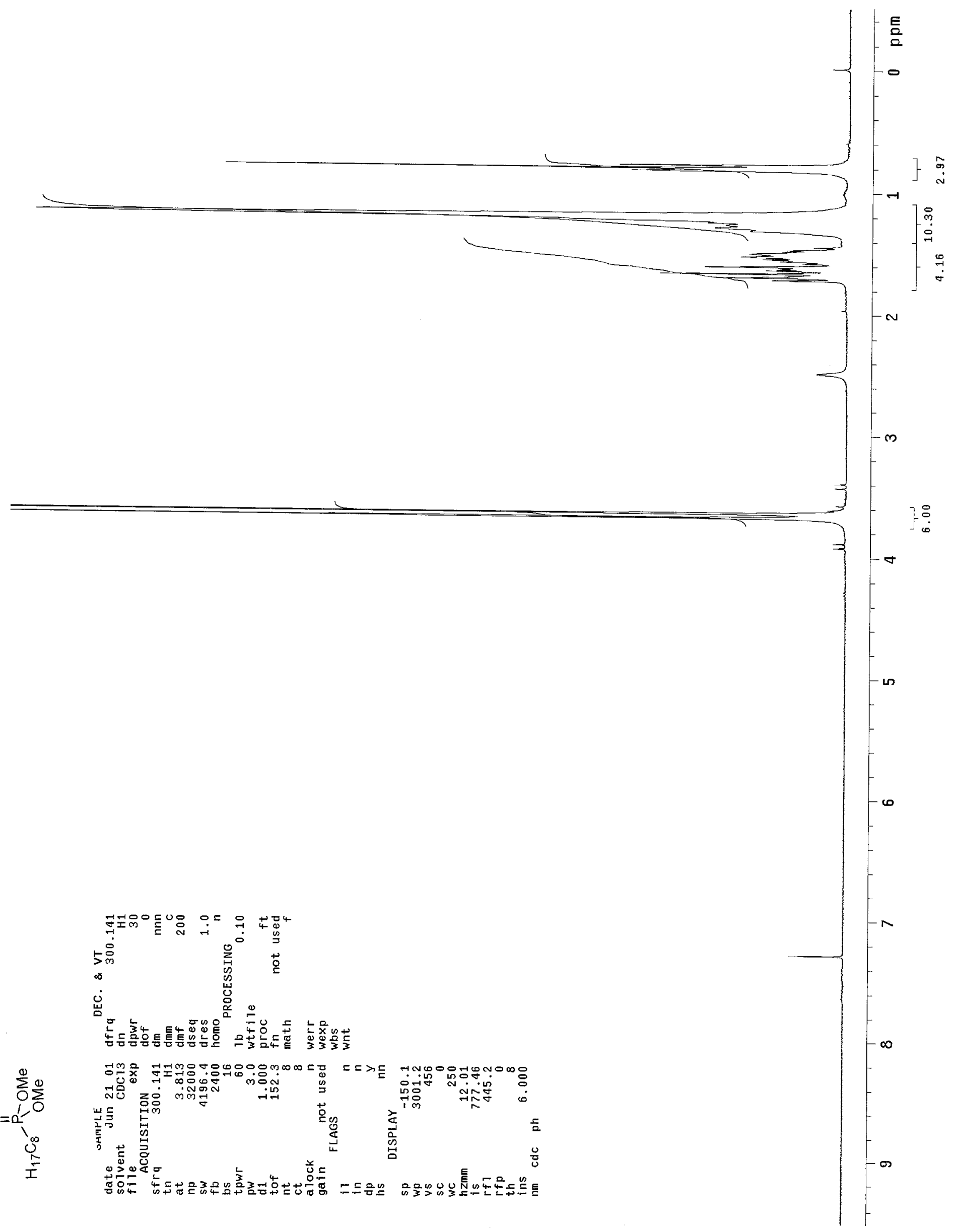




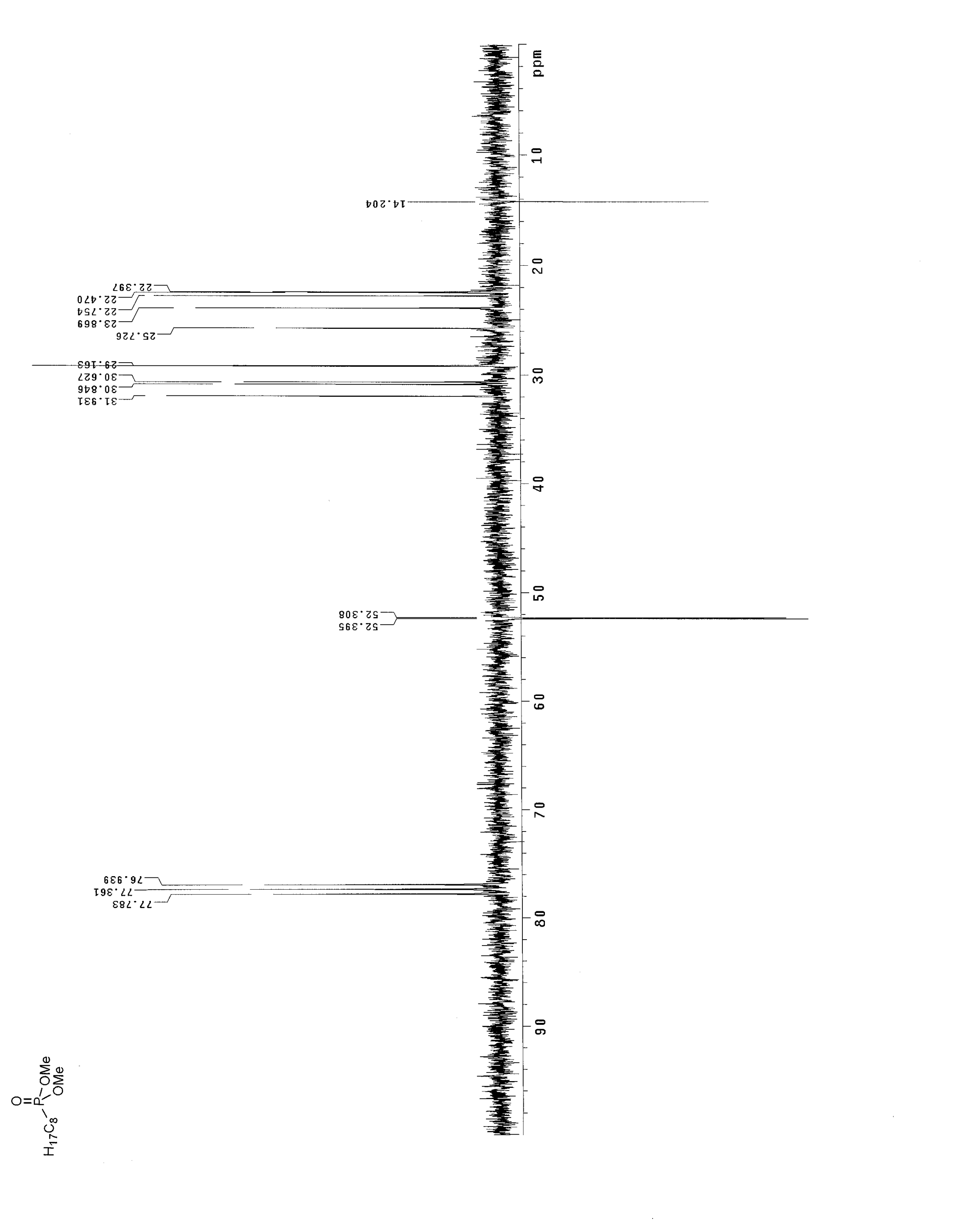




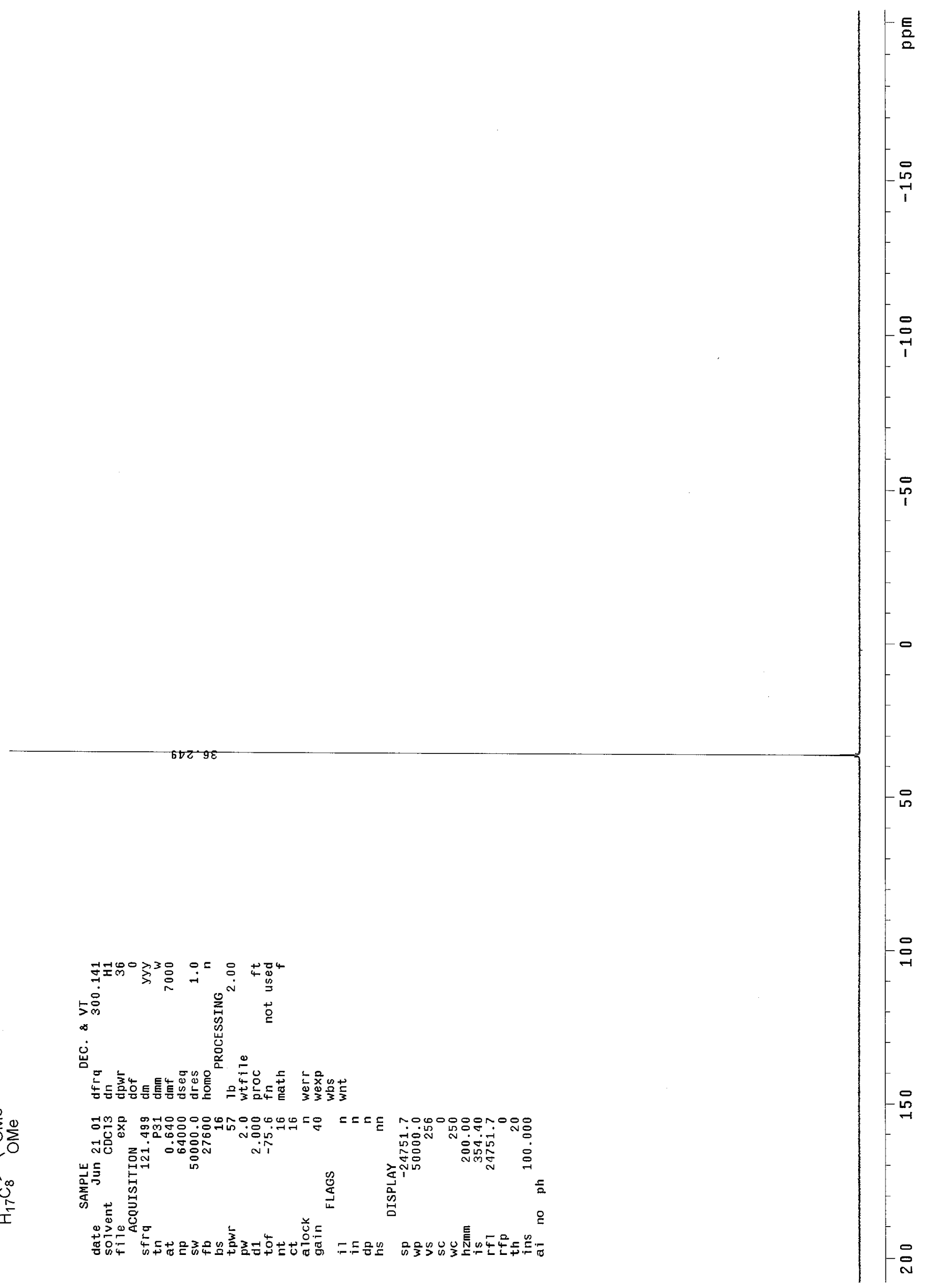




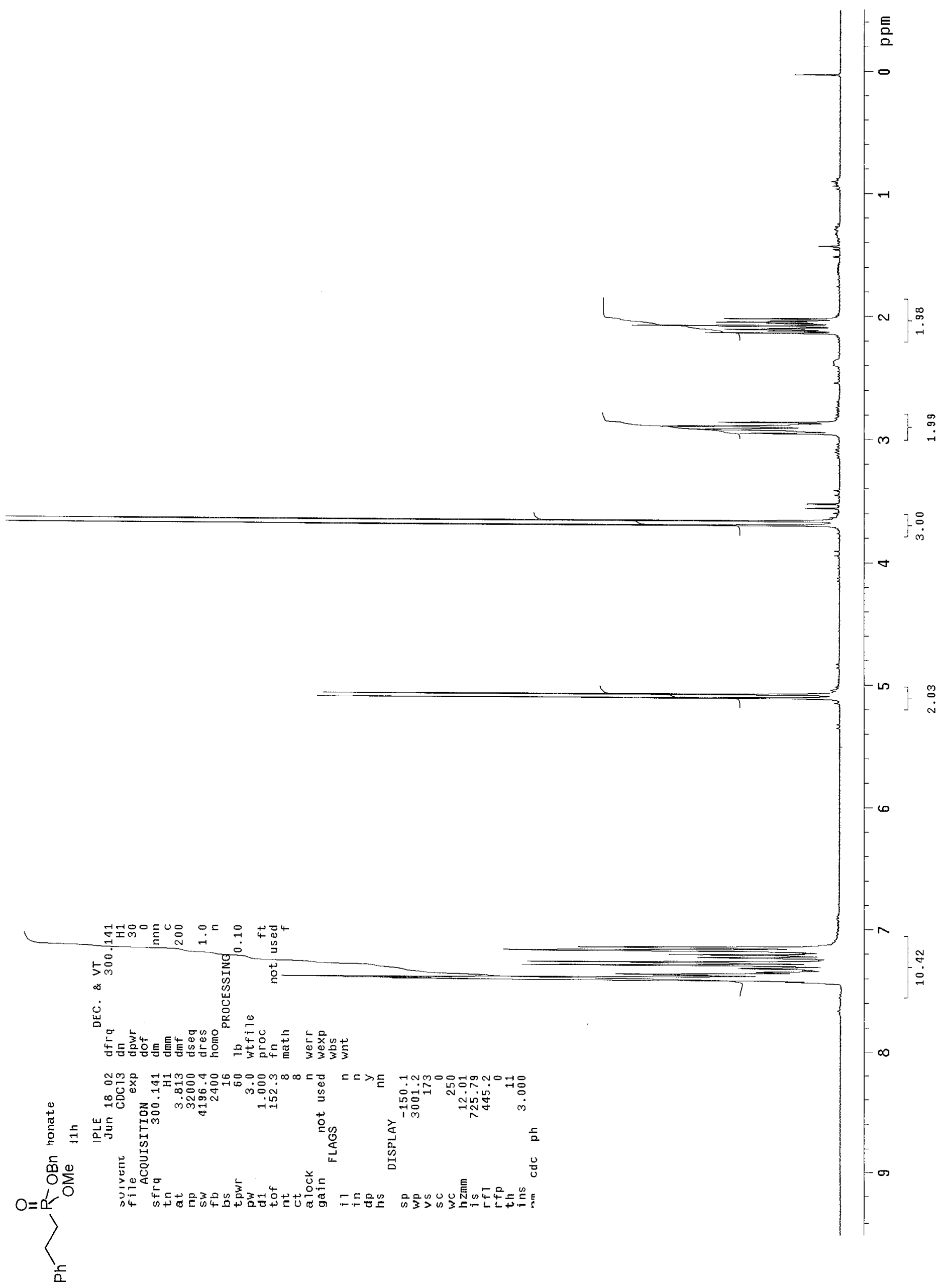




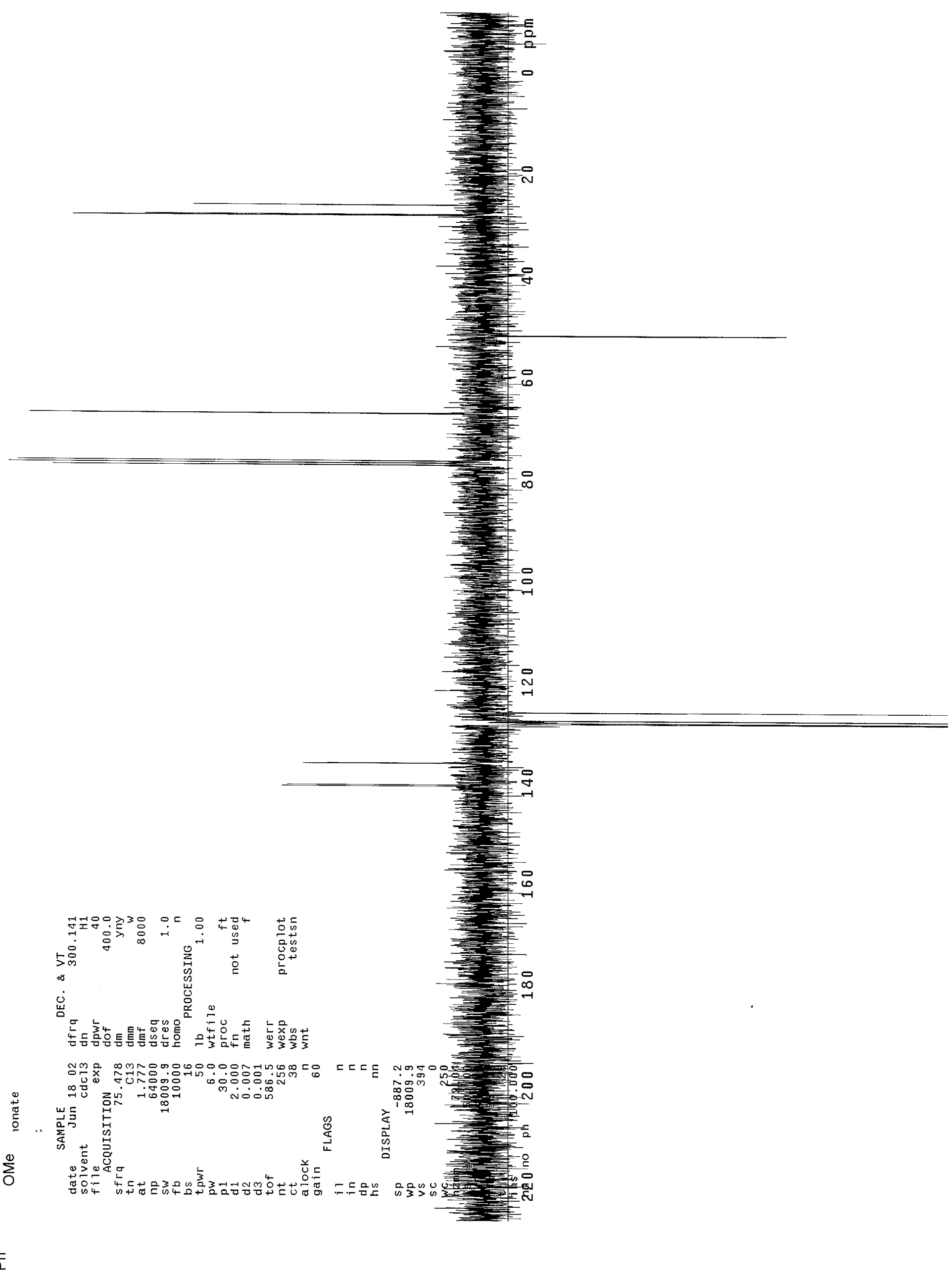




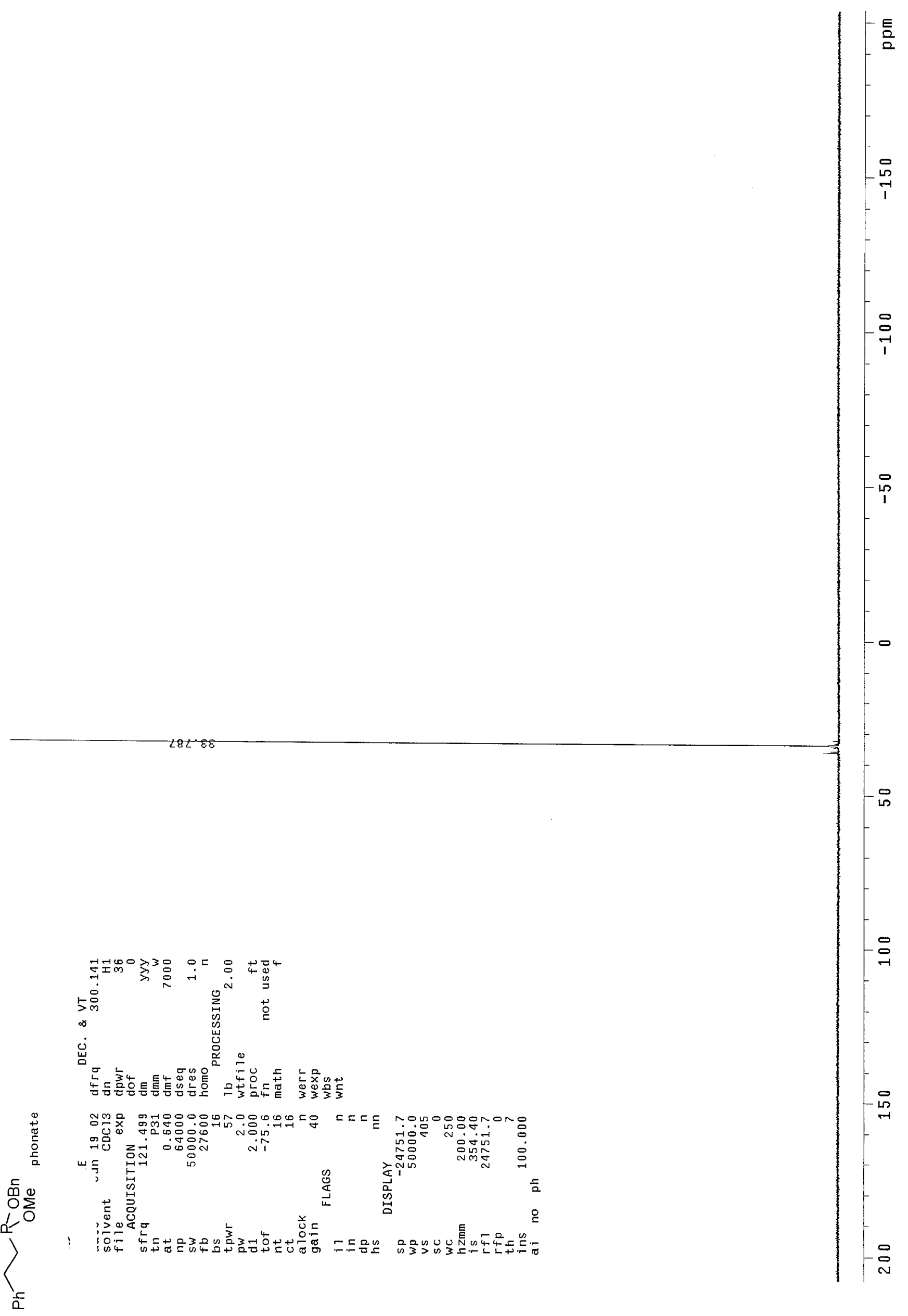




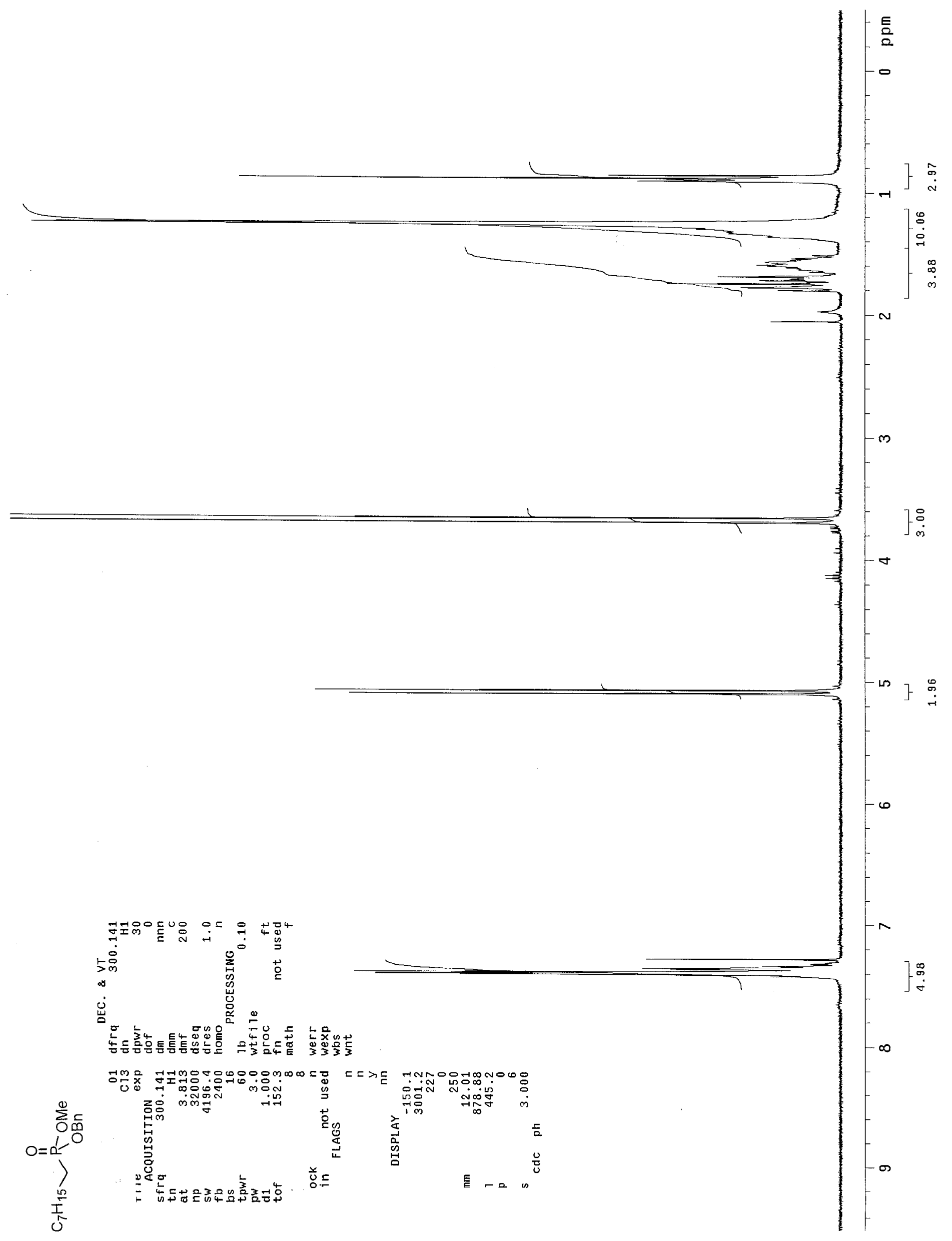




$$
1
$$




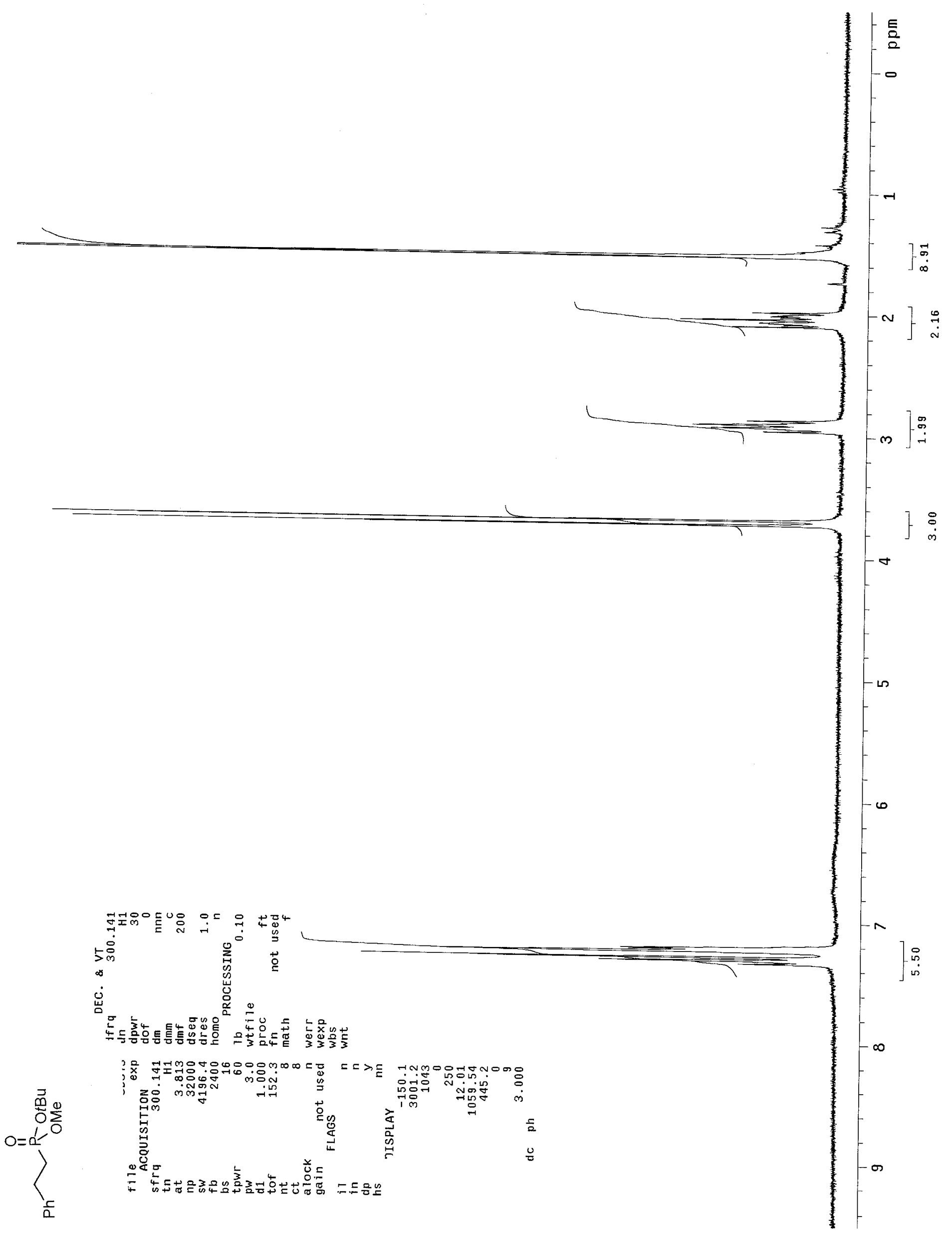




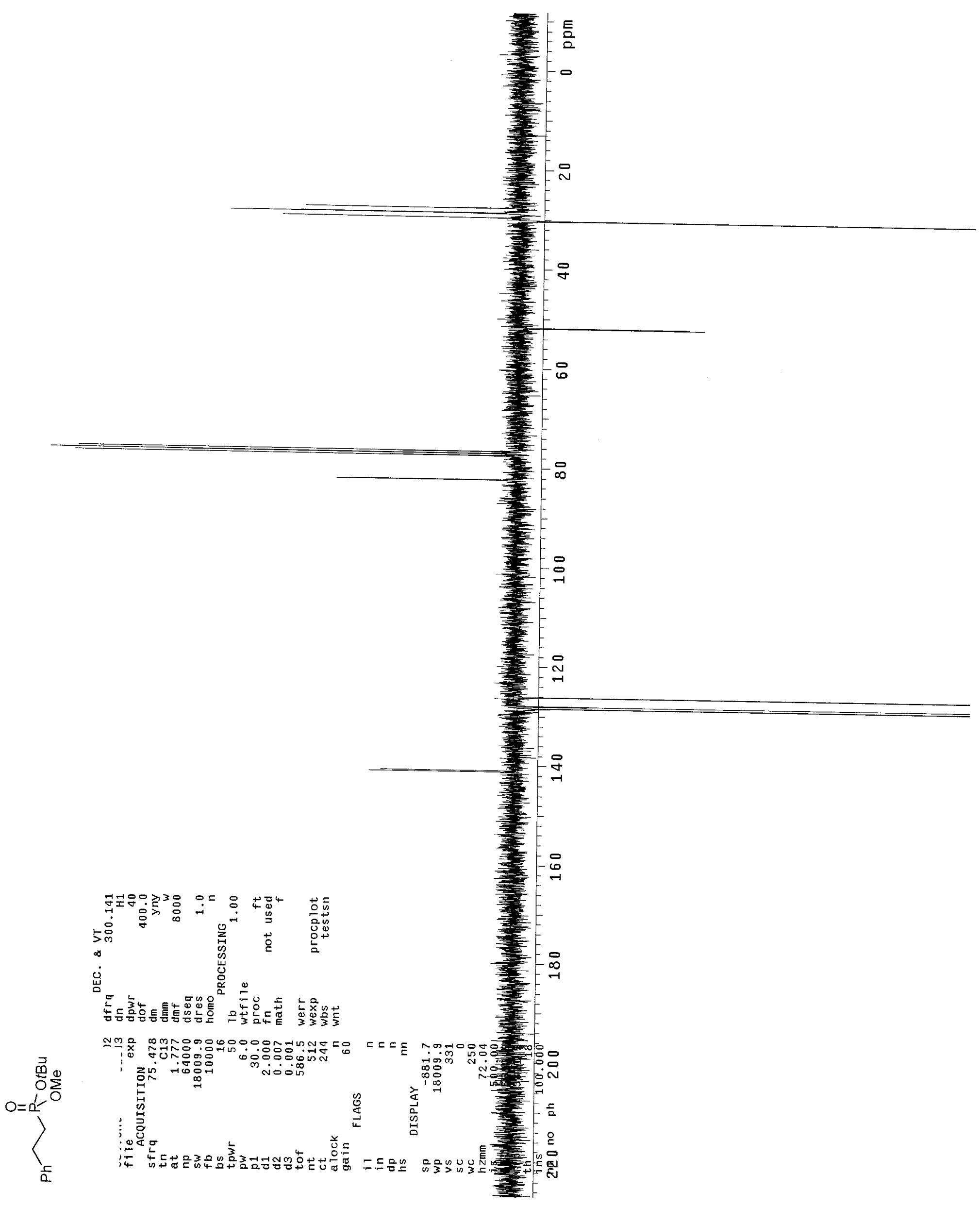




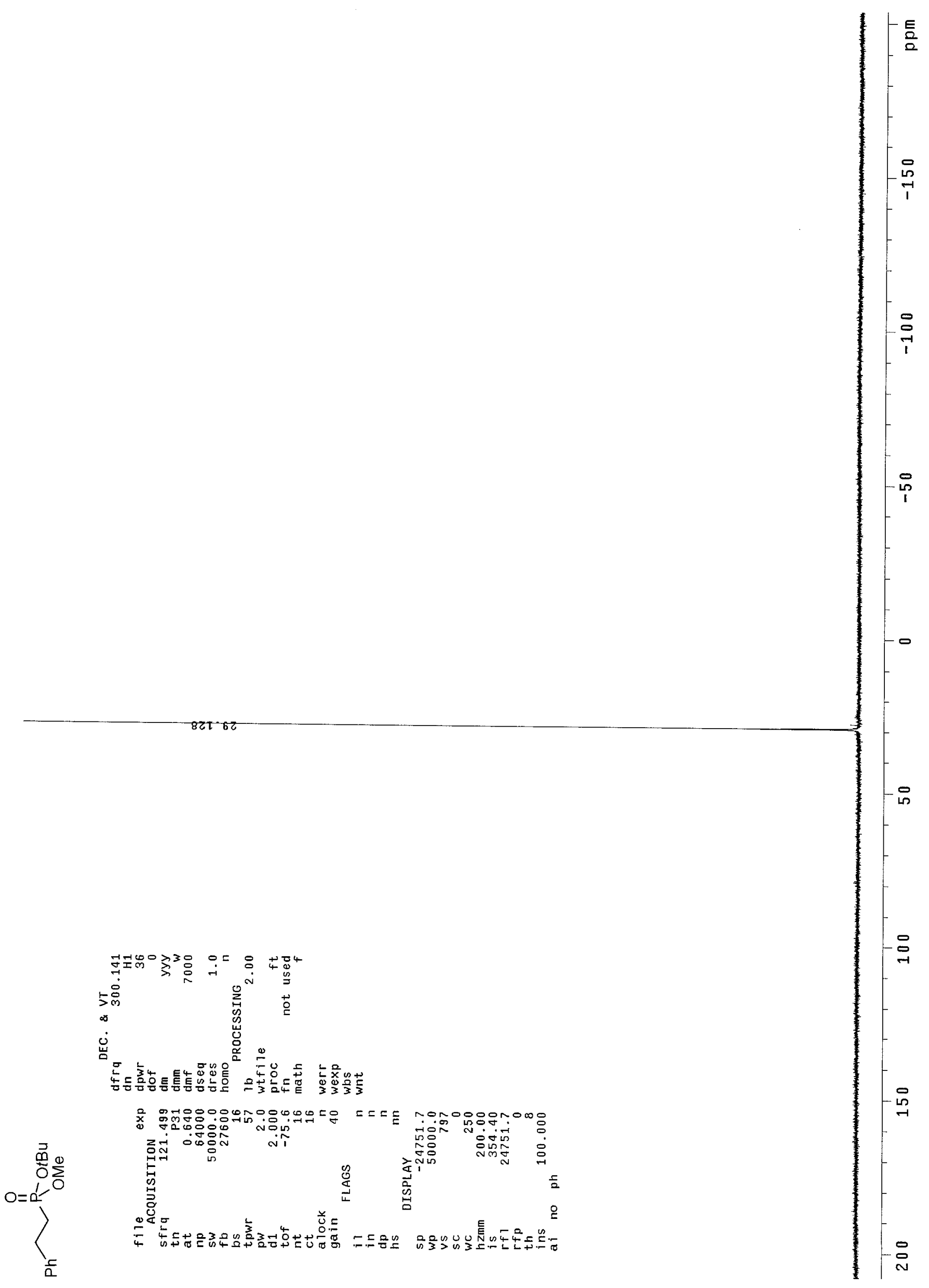




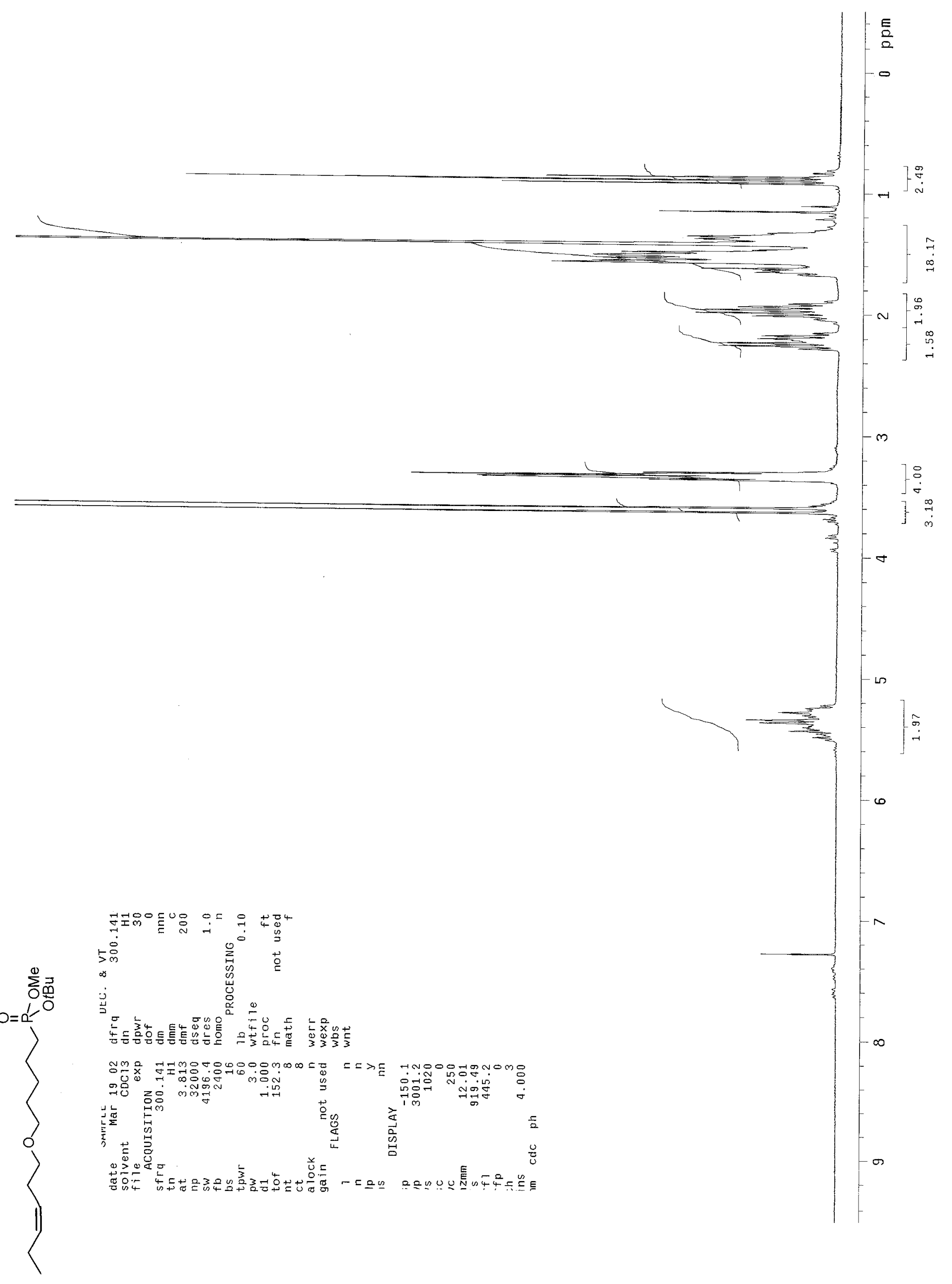




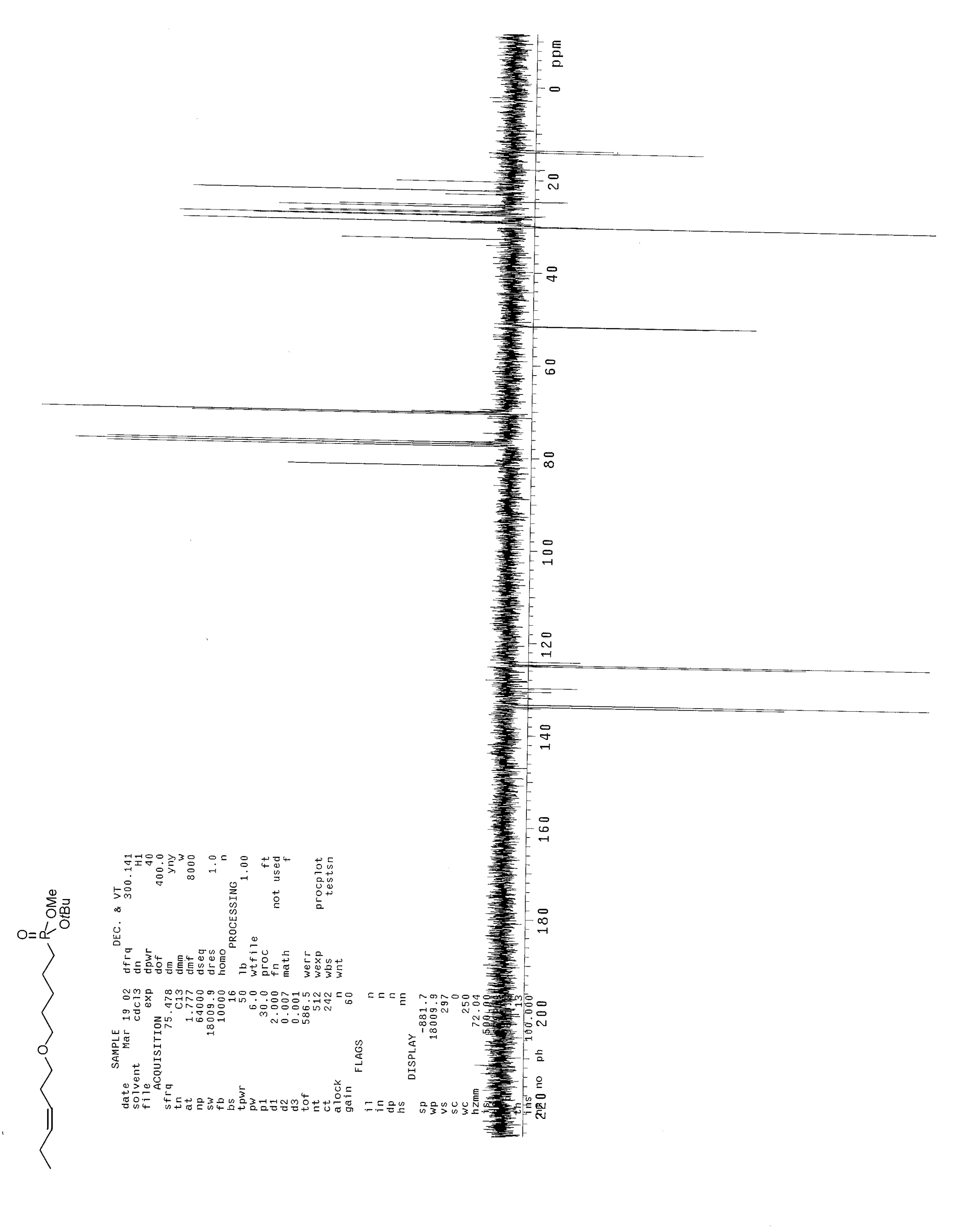




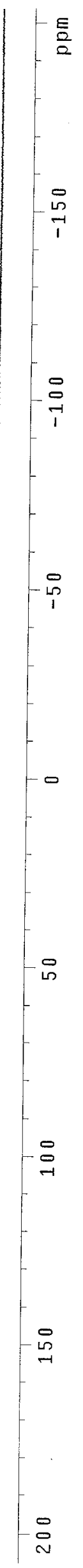




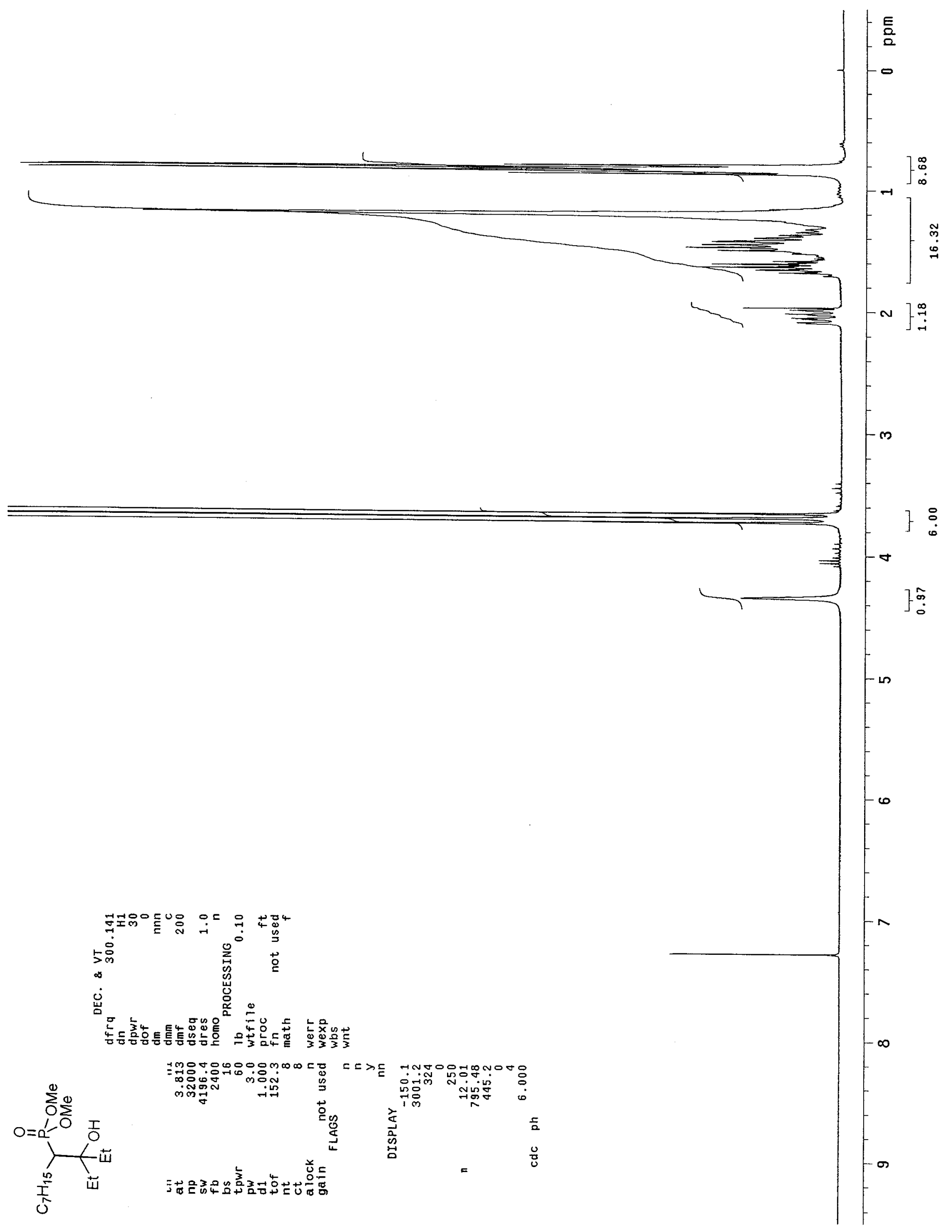




$$
1
$$




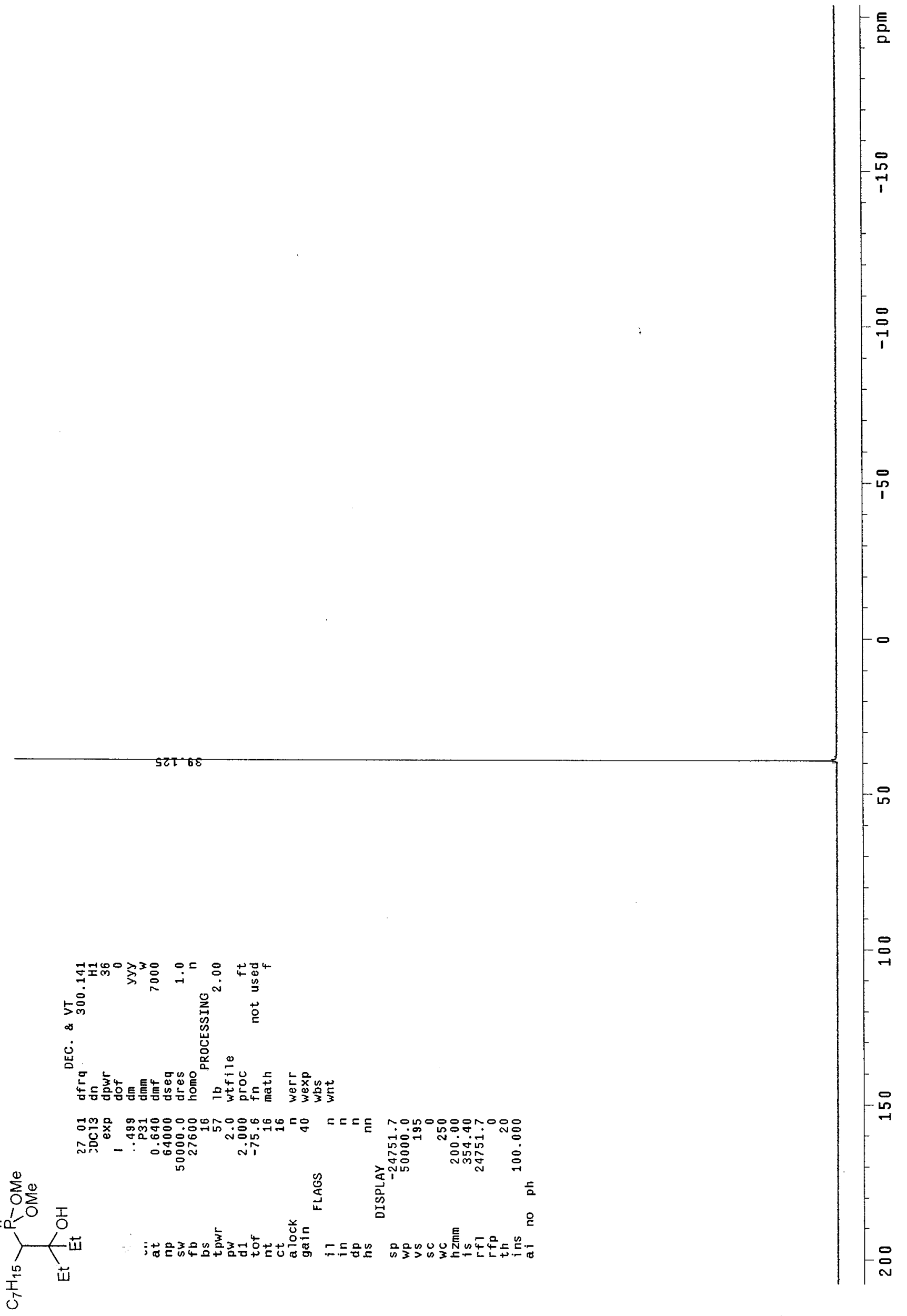




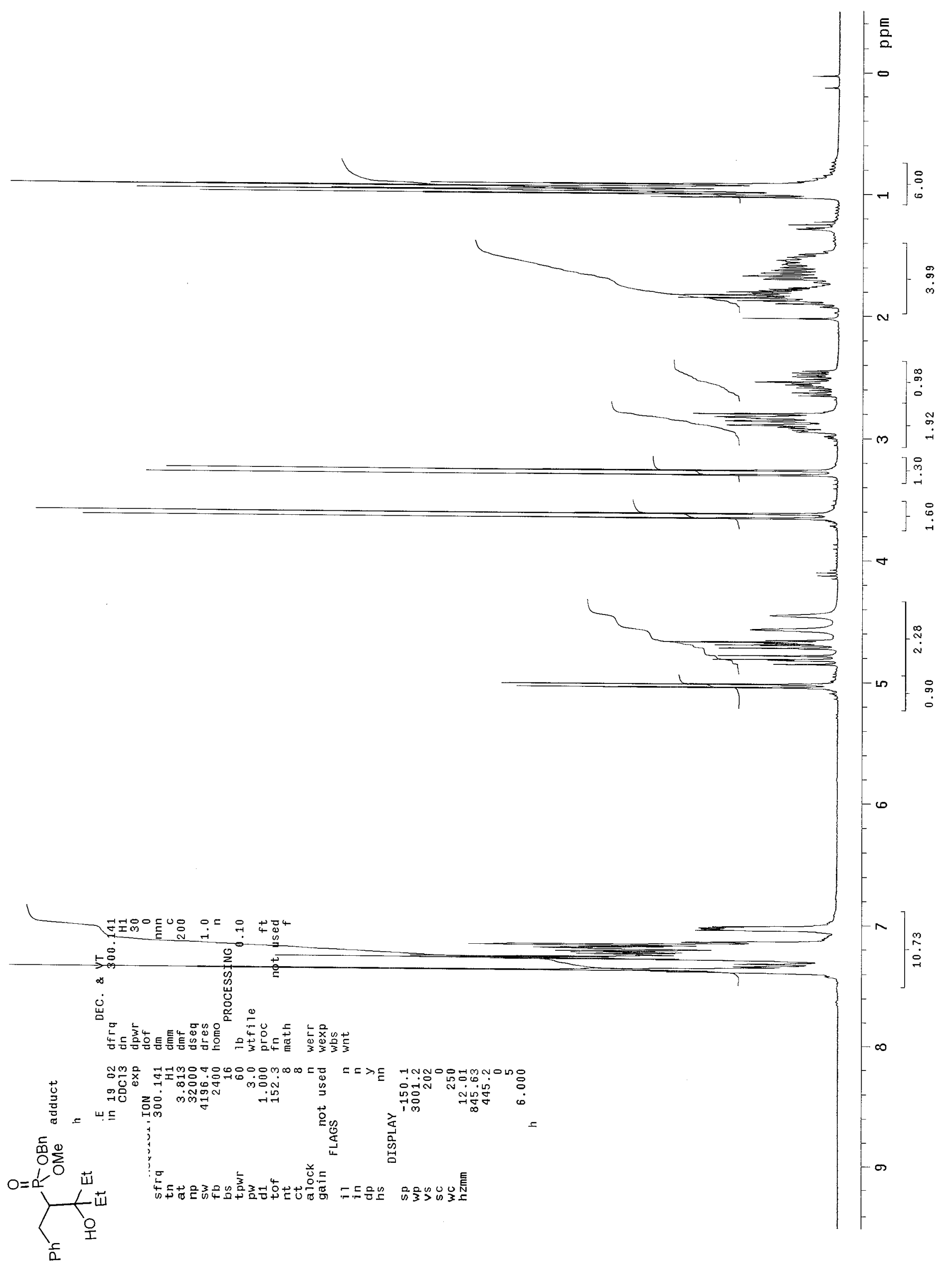




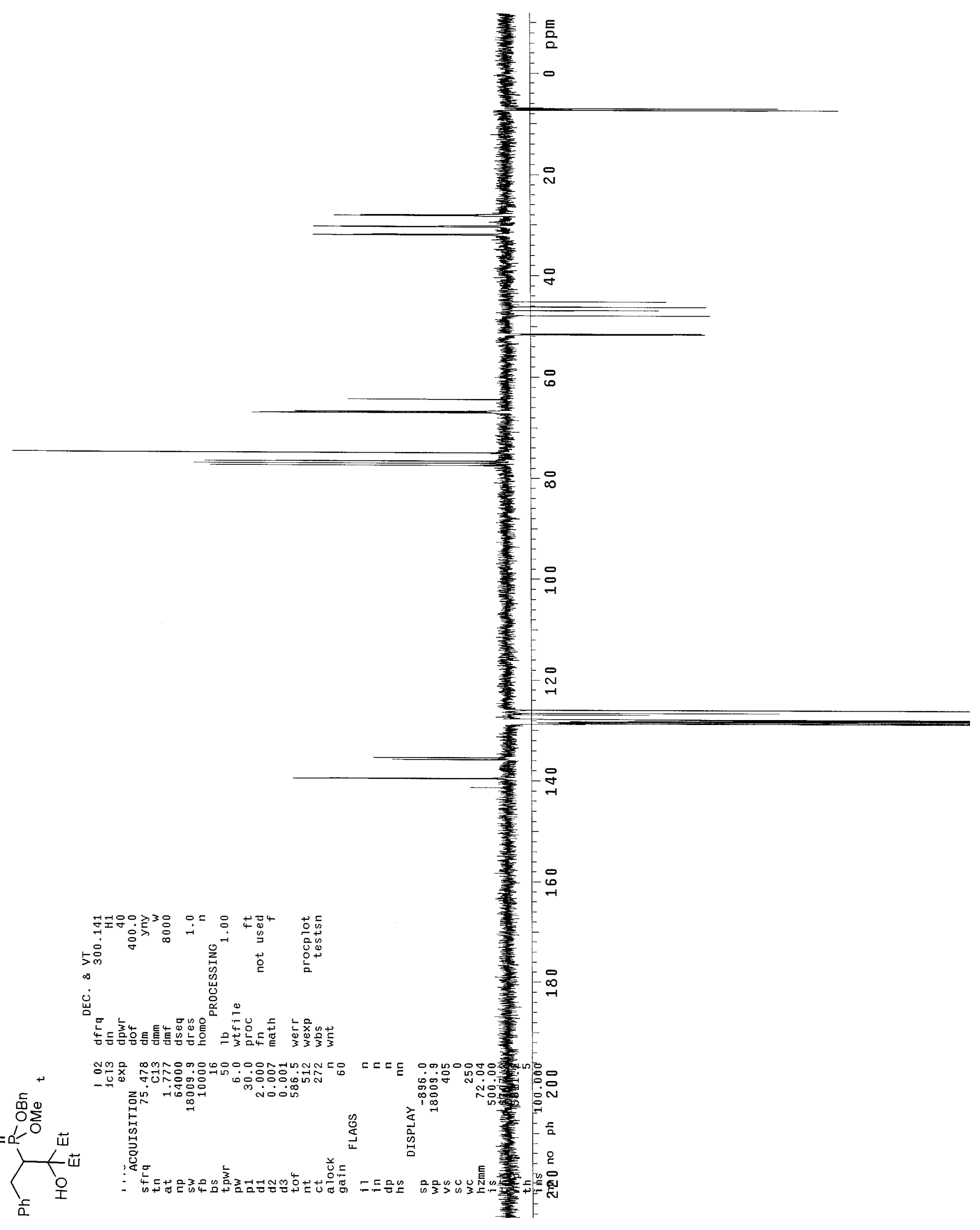




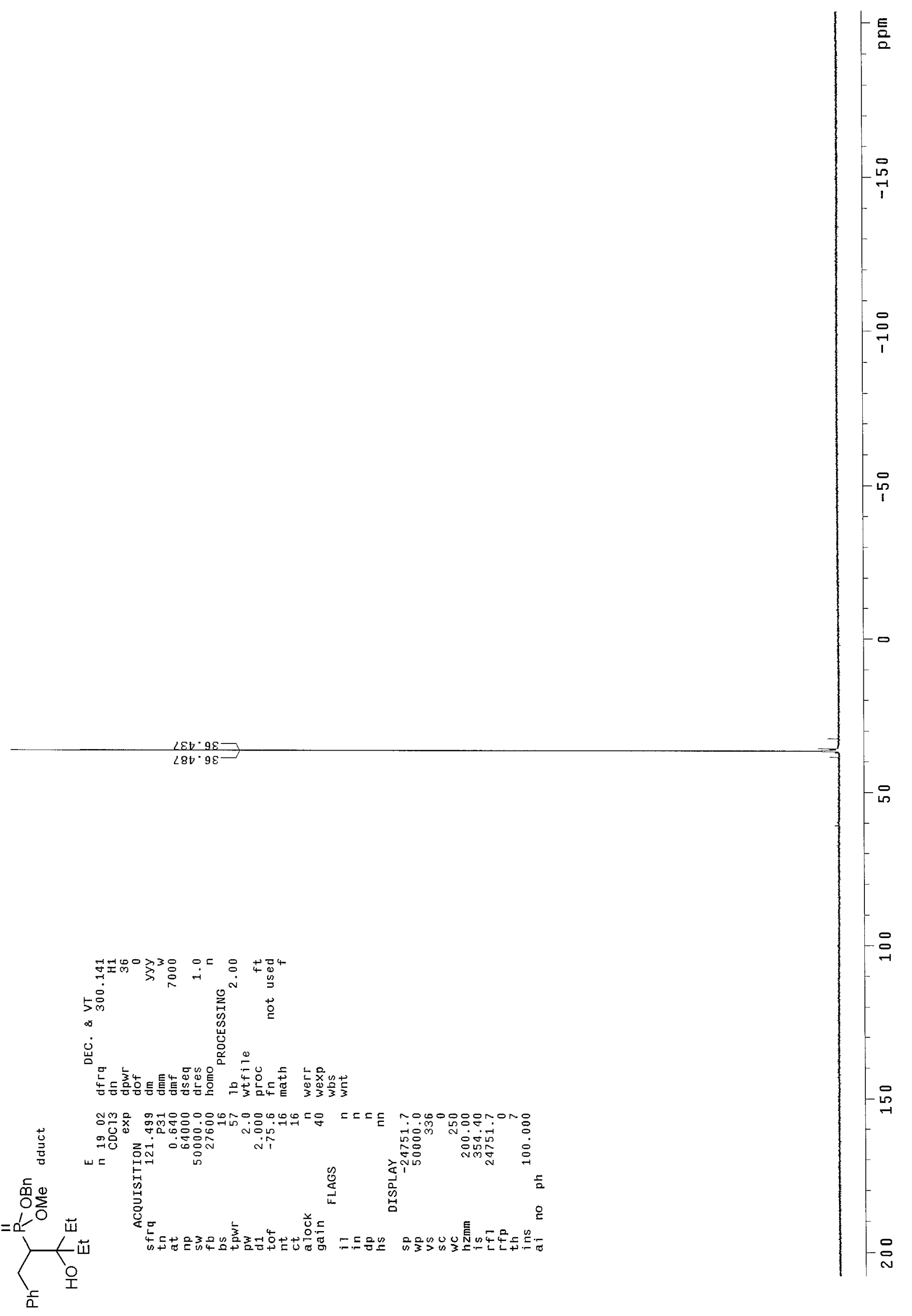





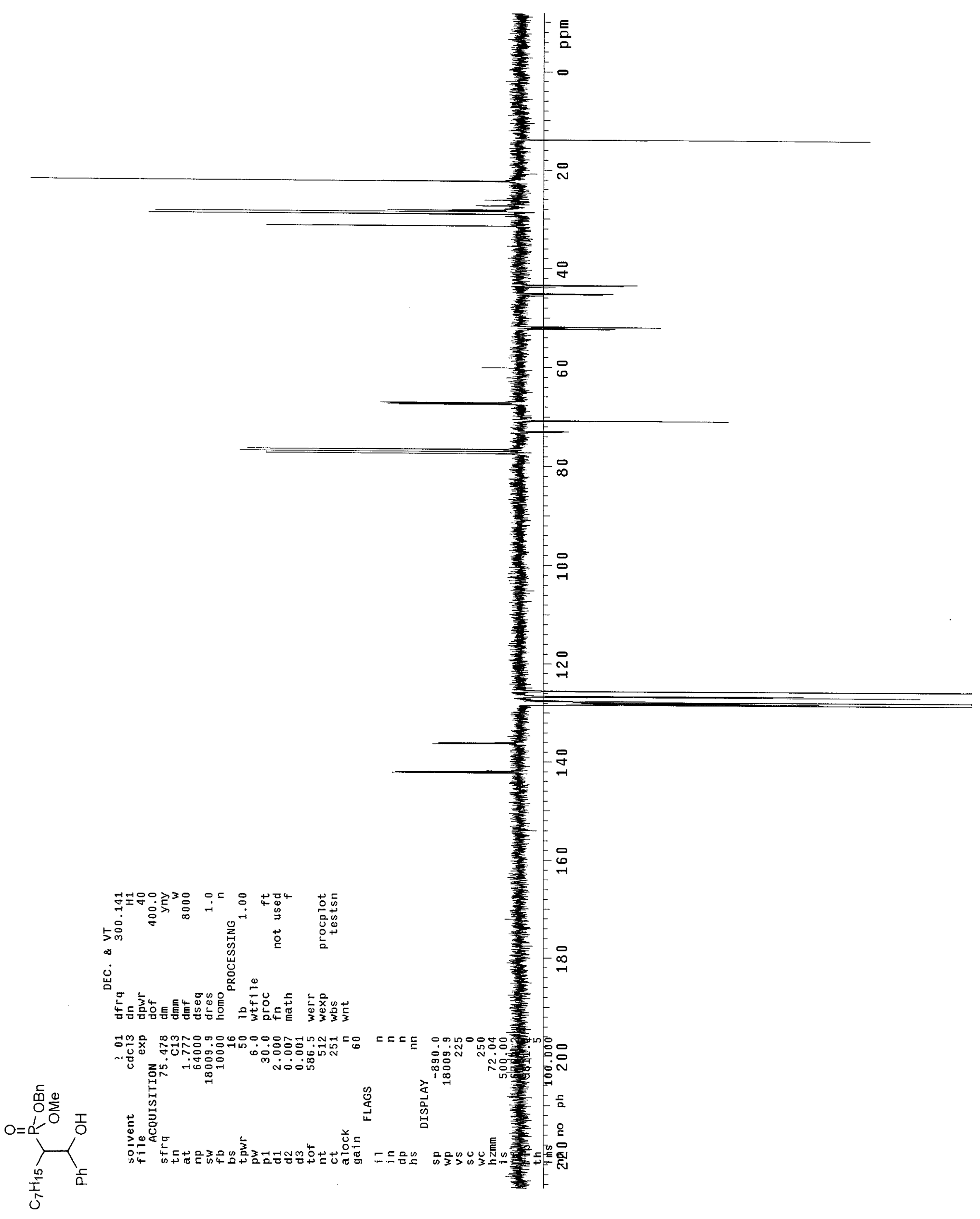




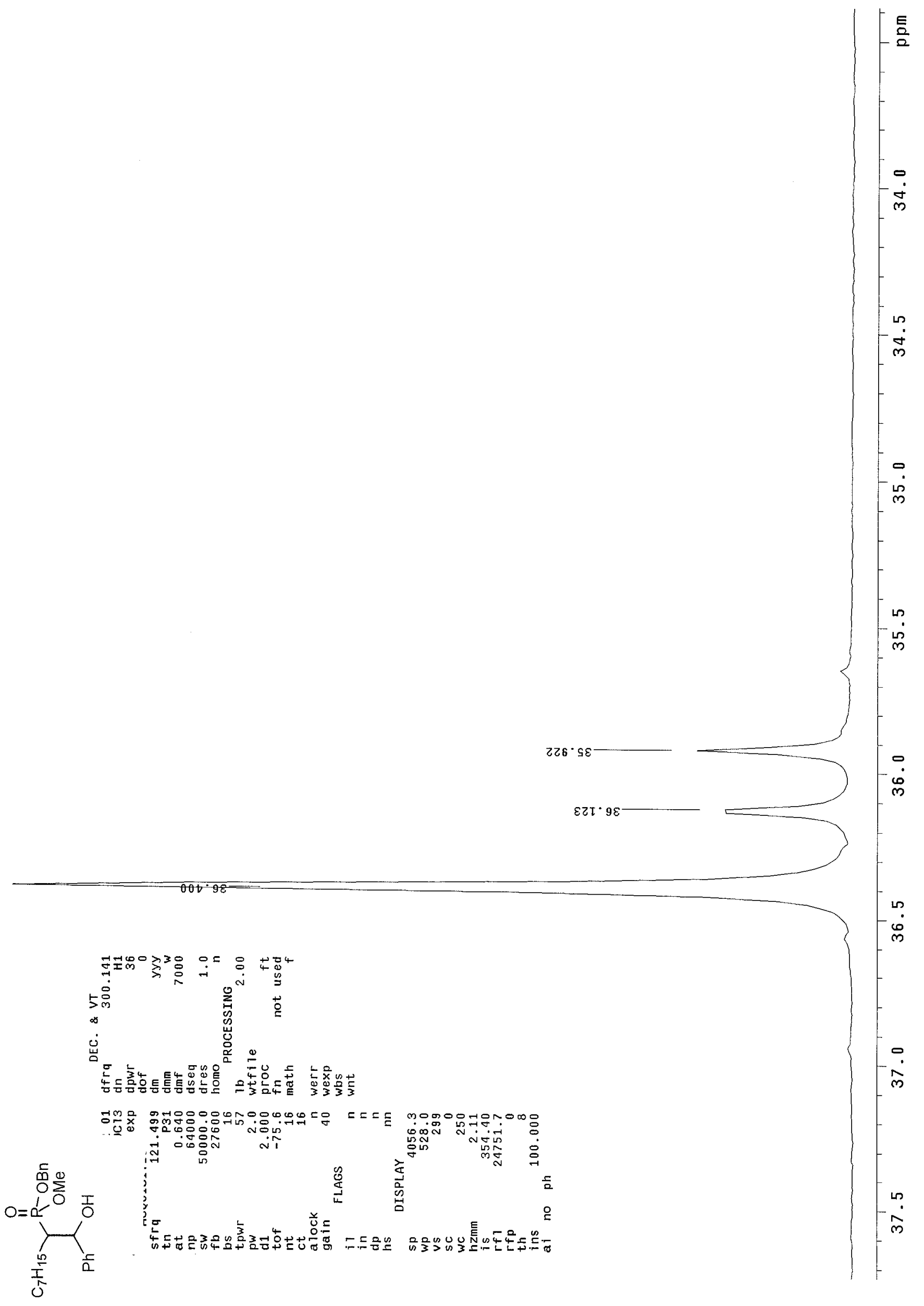




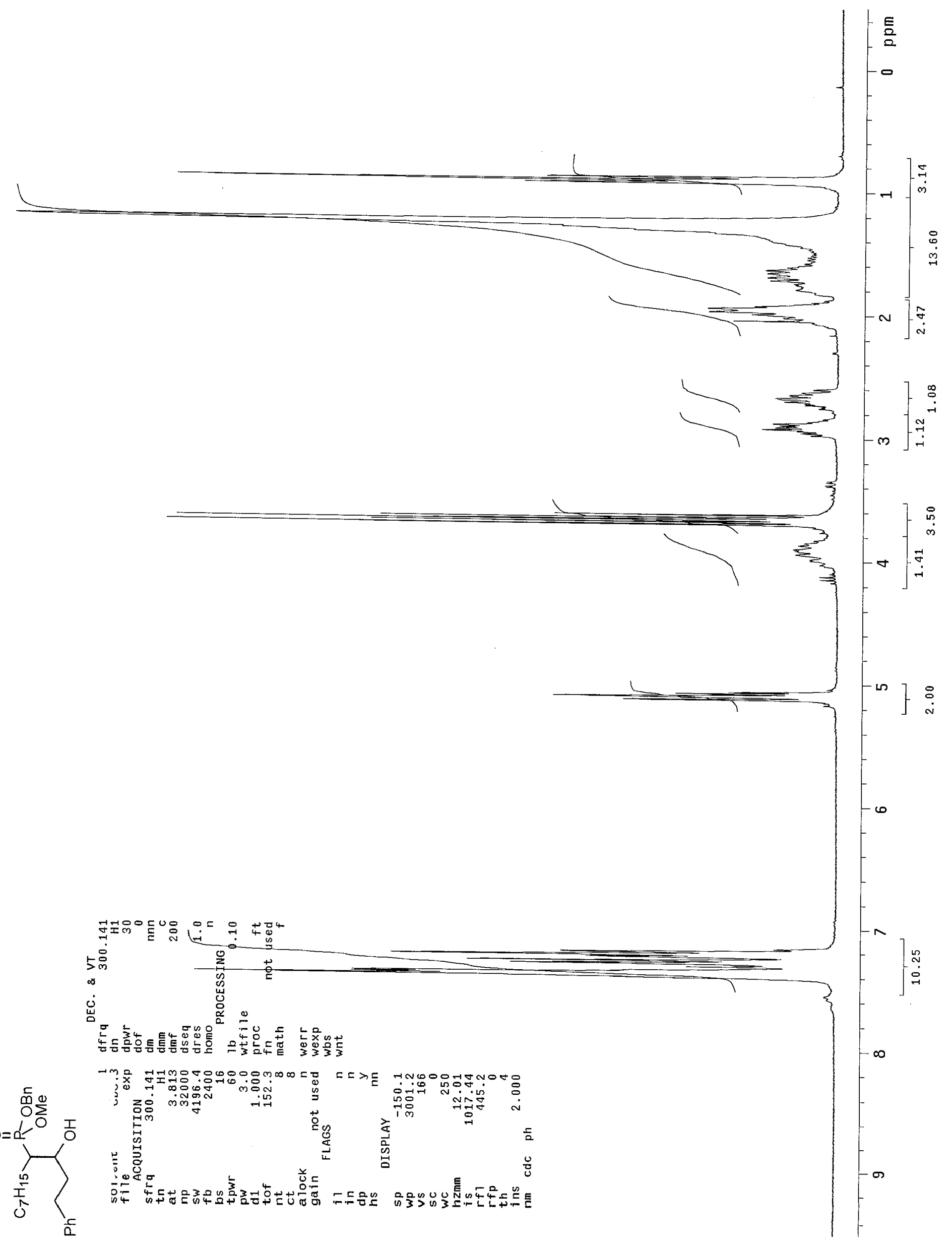




$$
\text { 春 }
$$




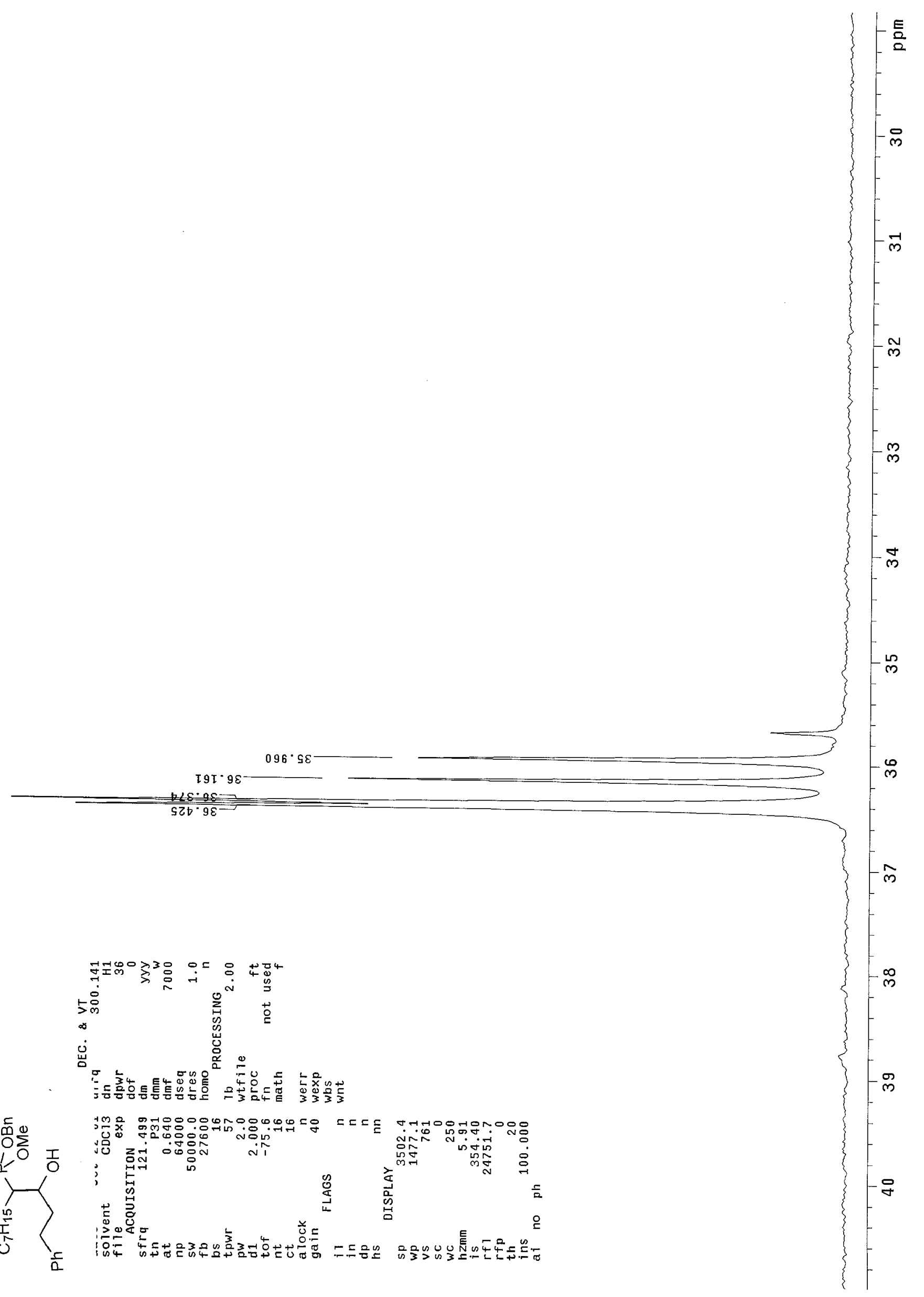




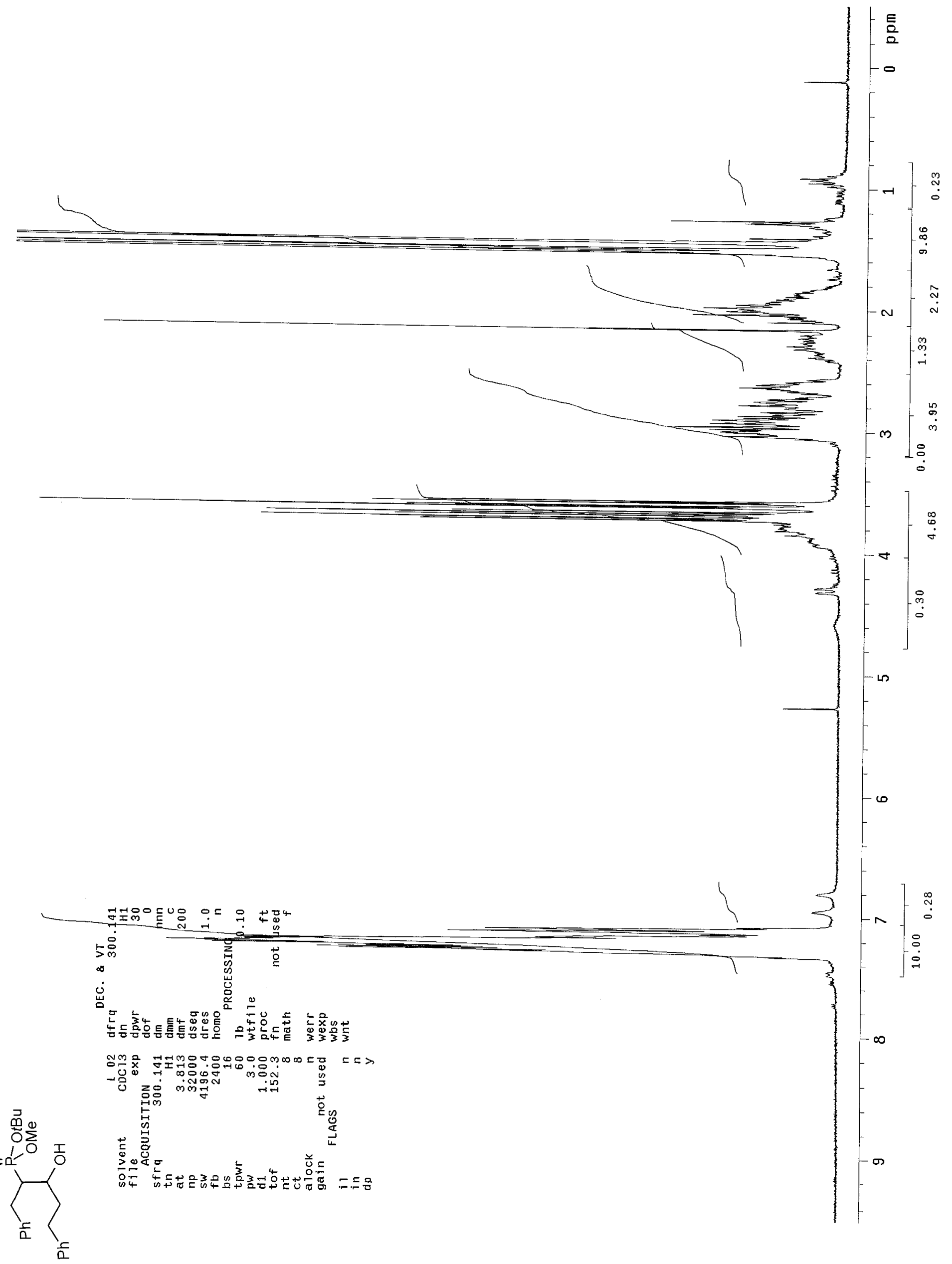




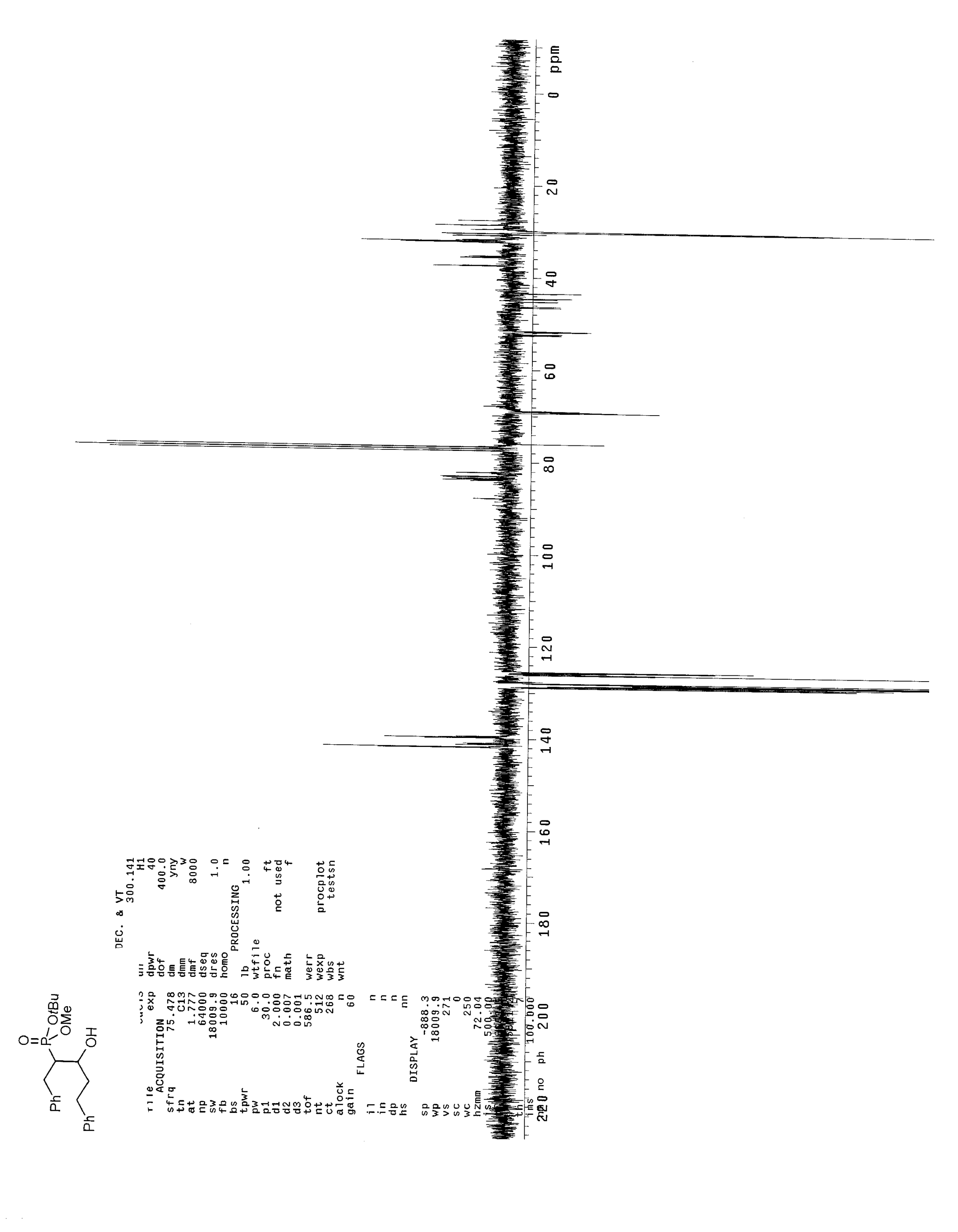




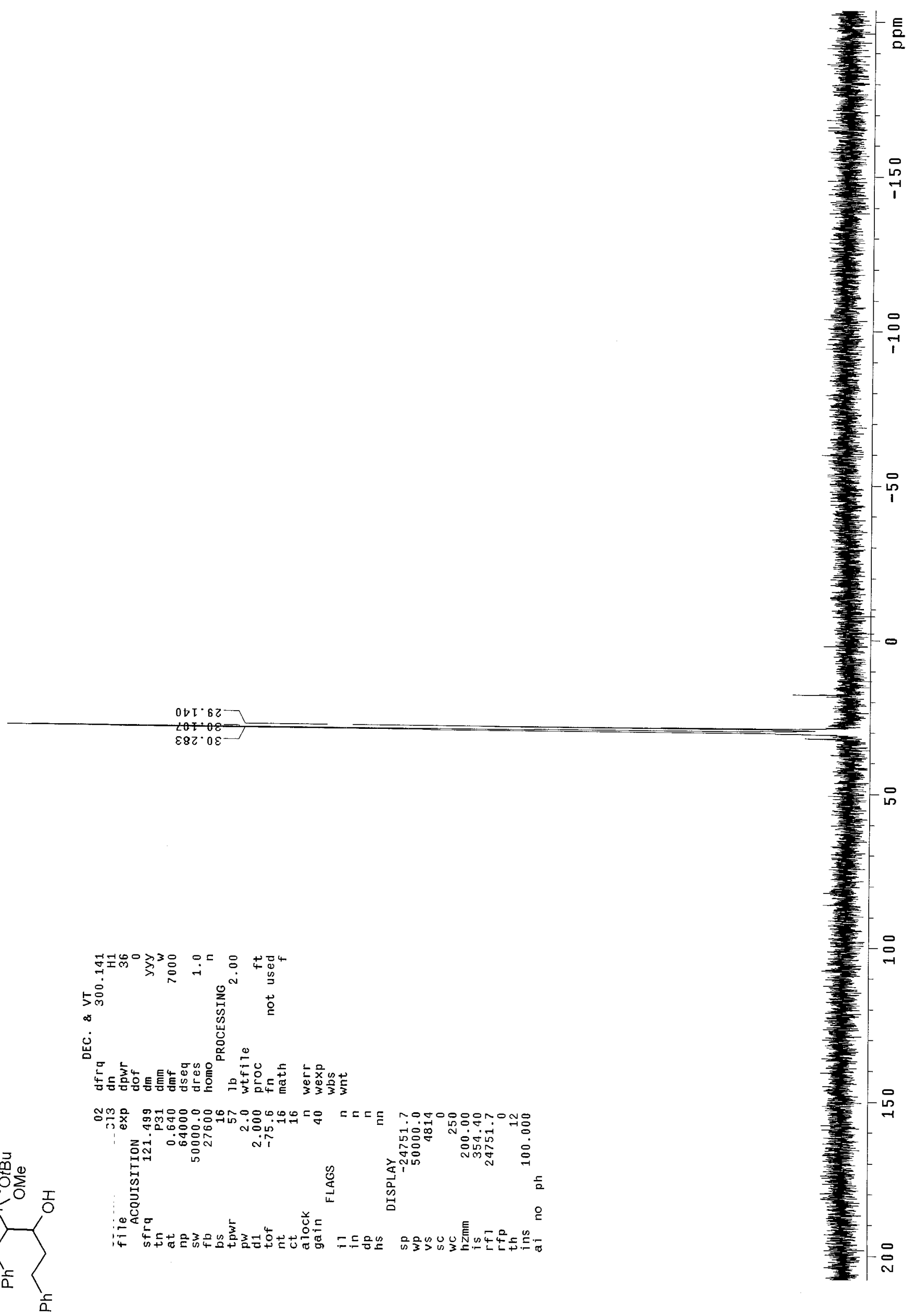




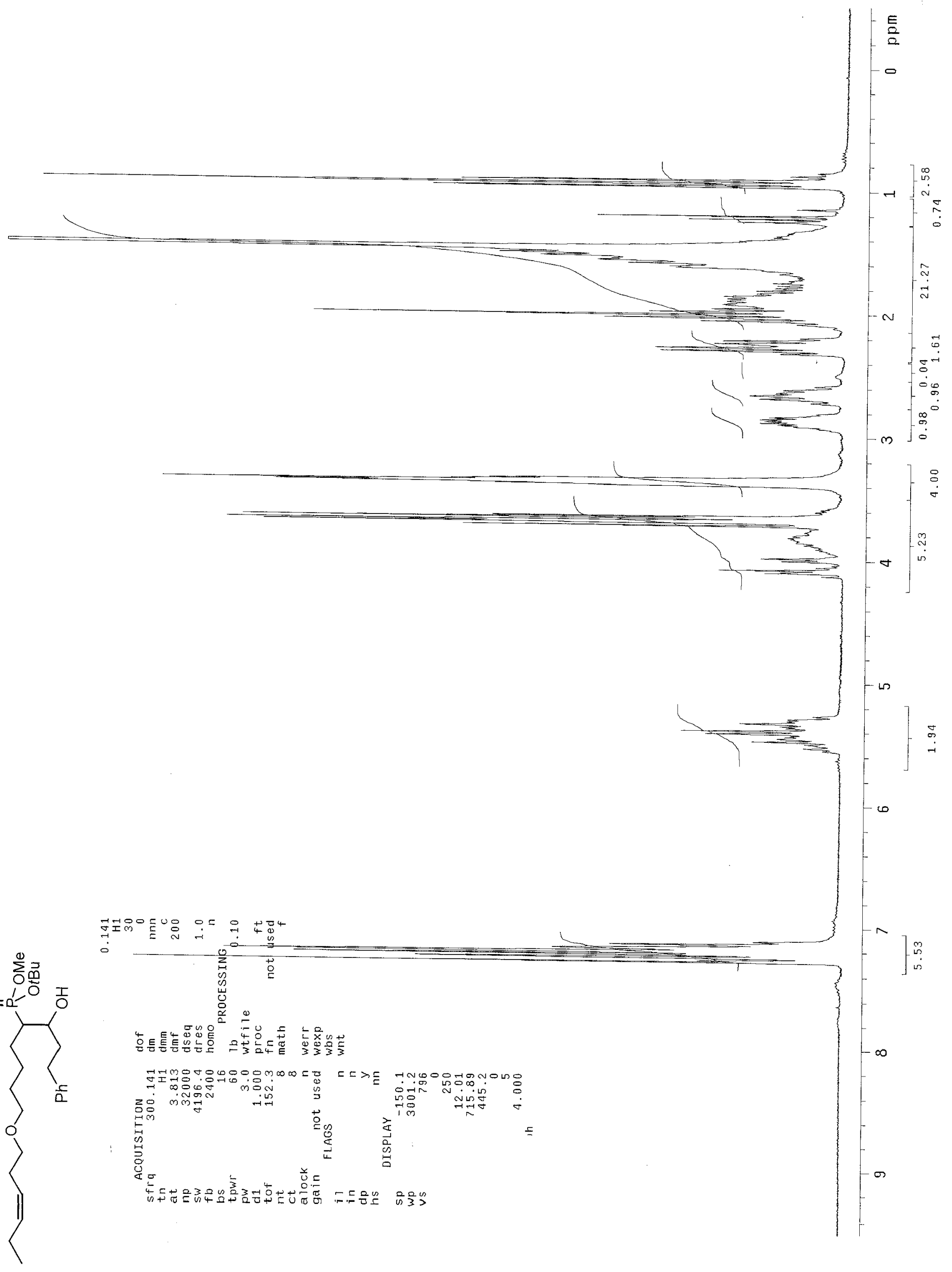




$$
1
$$




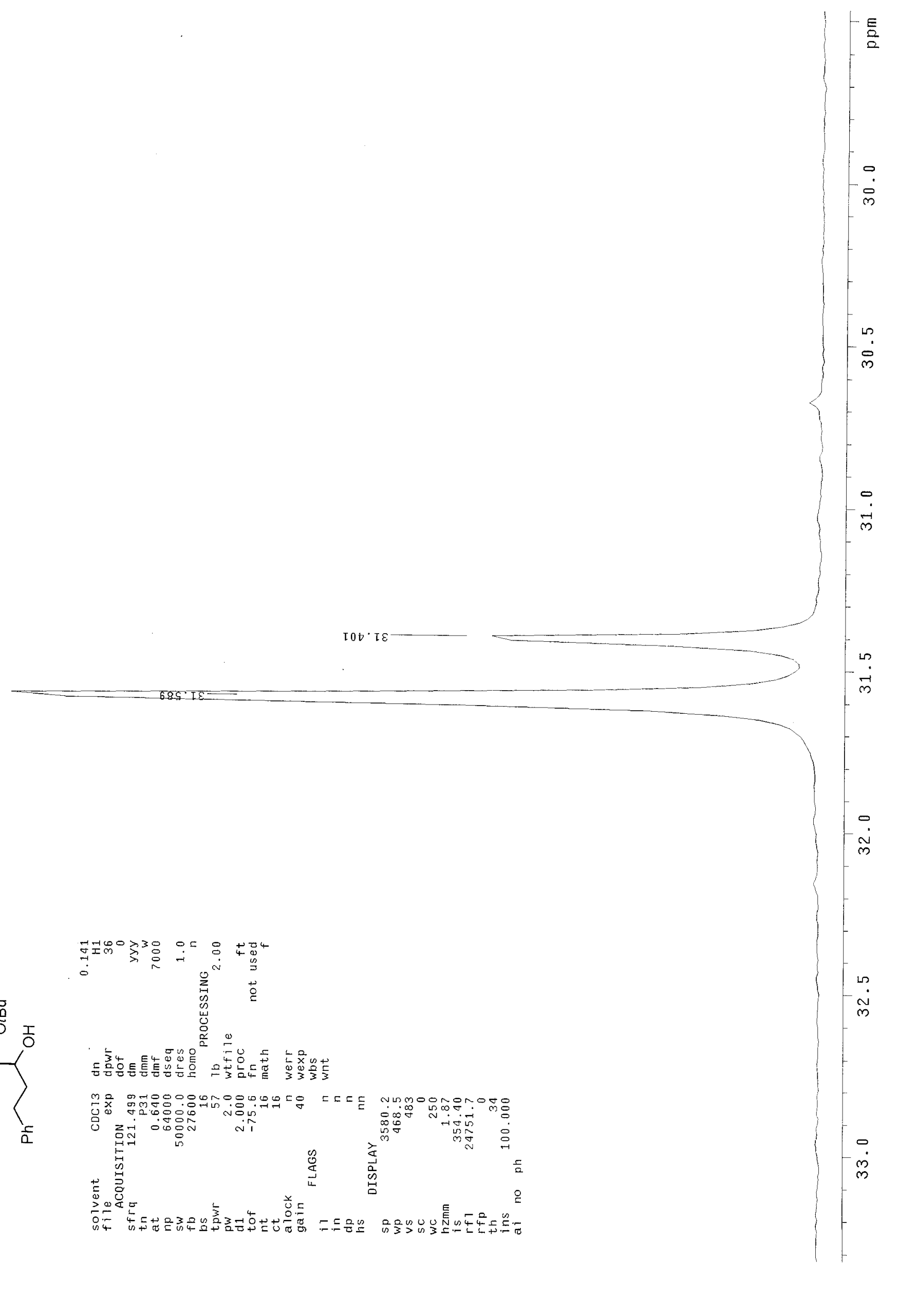




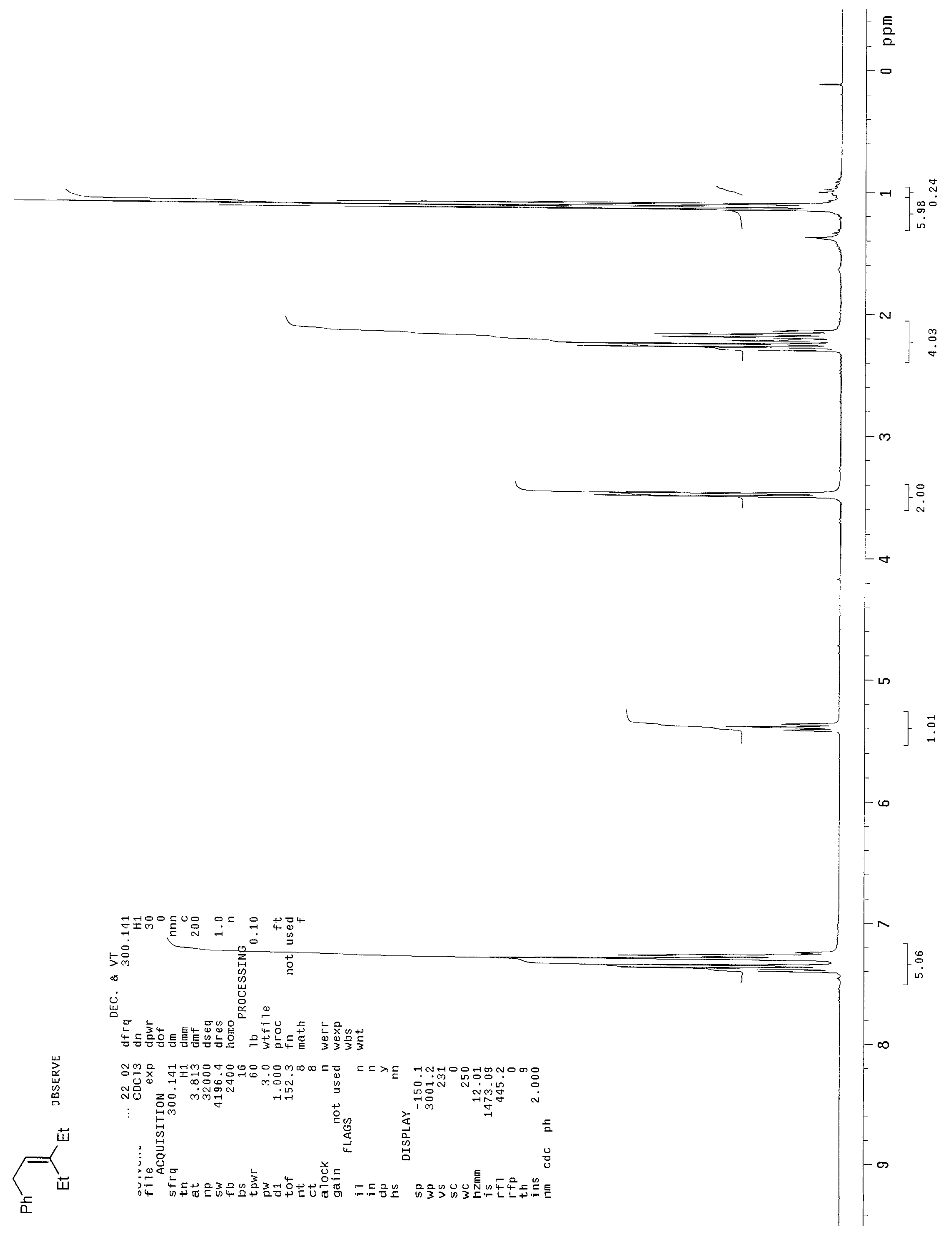




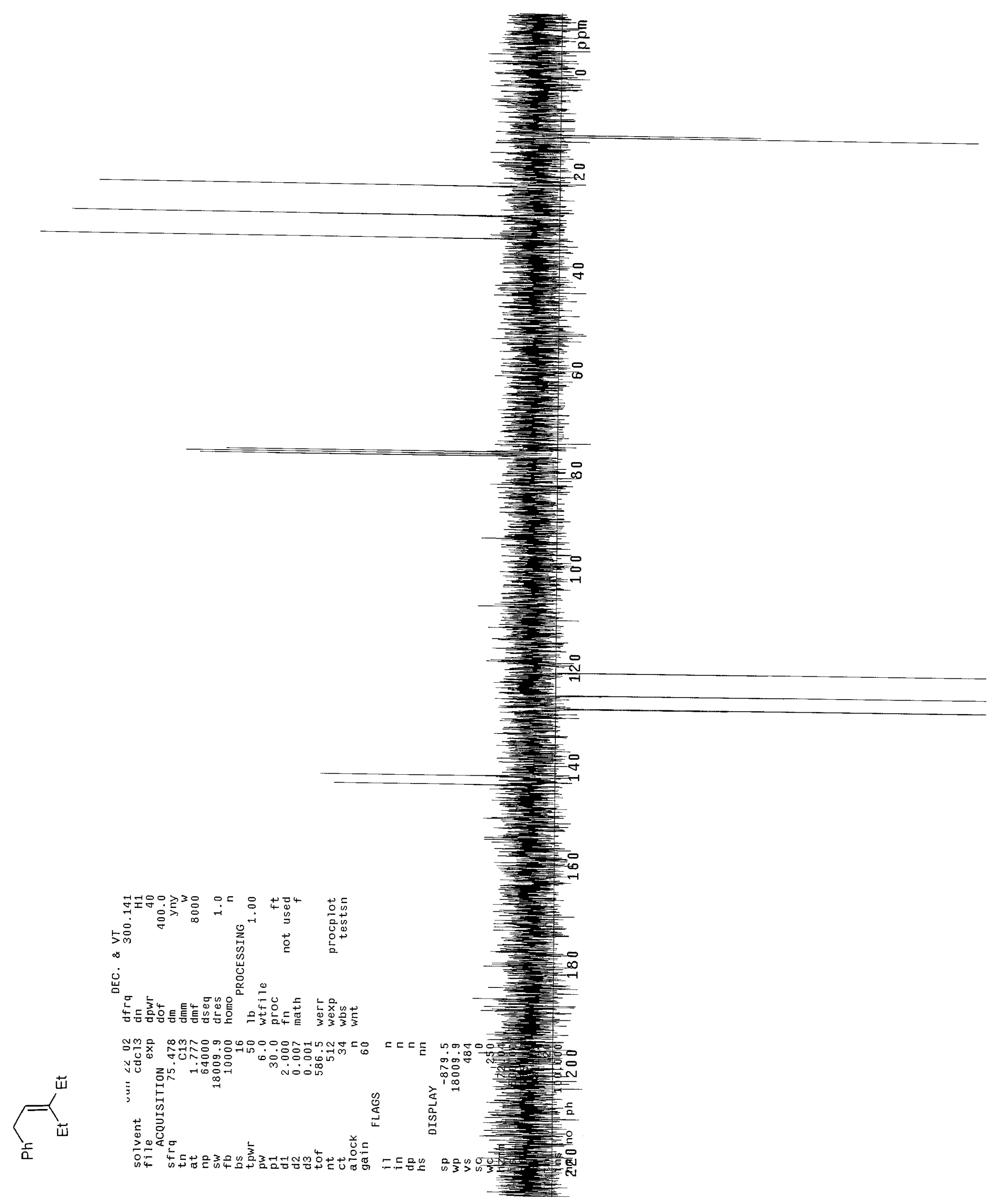




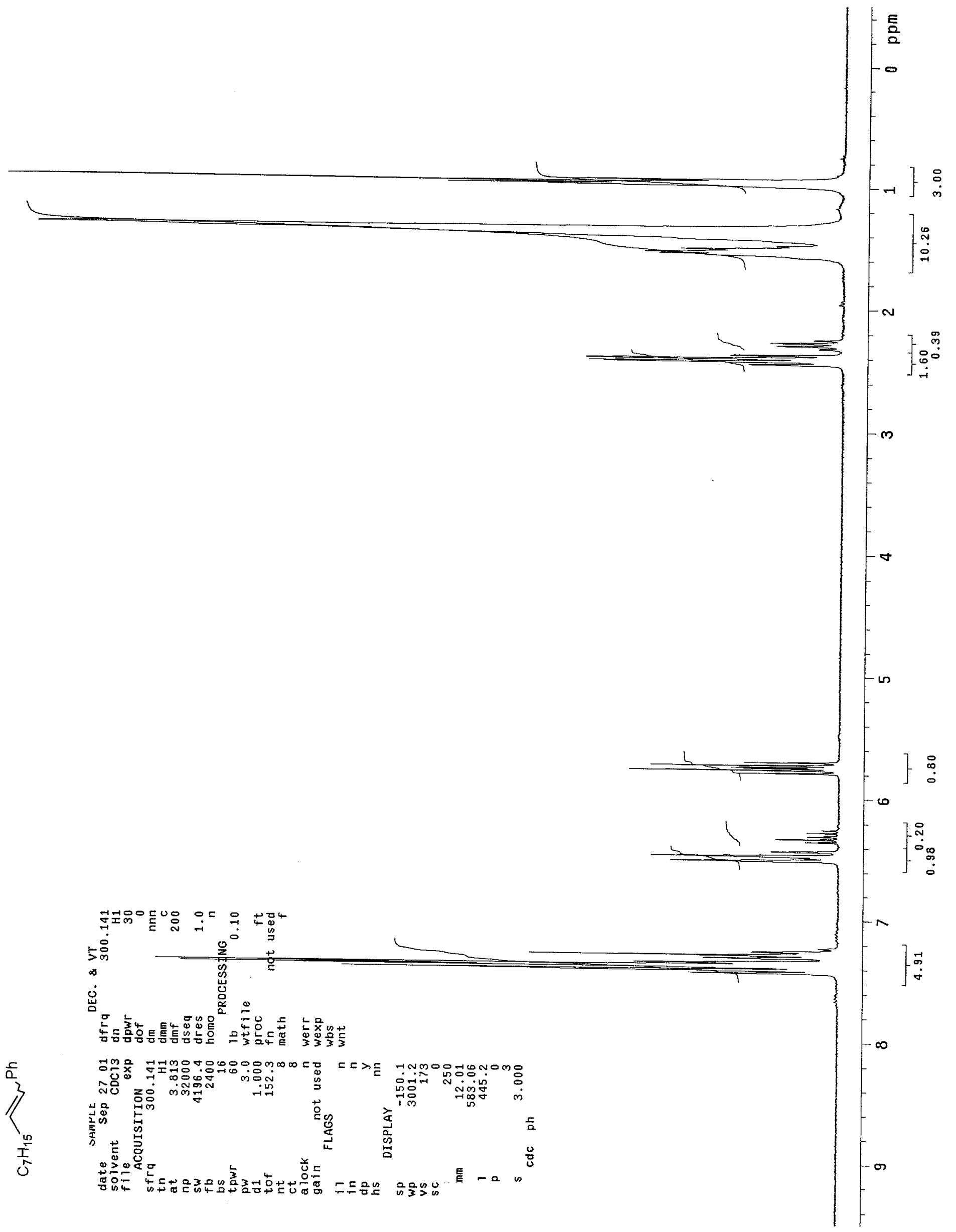




$$
\neq
$$




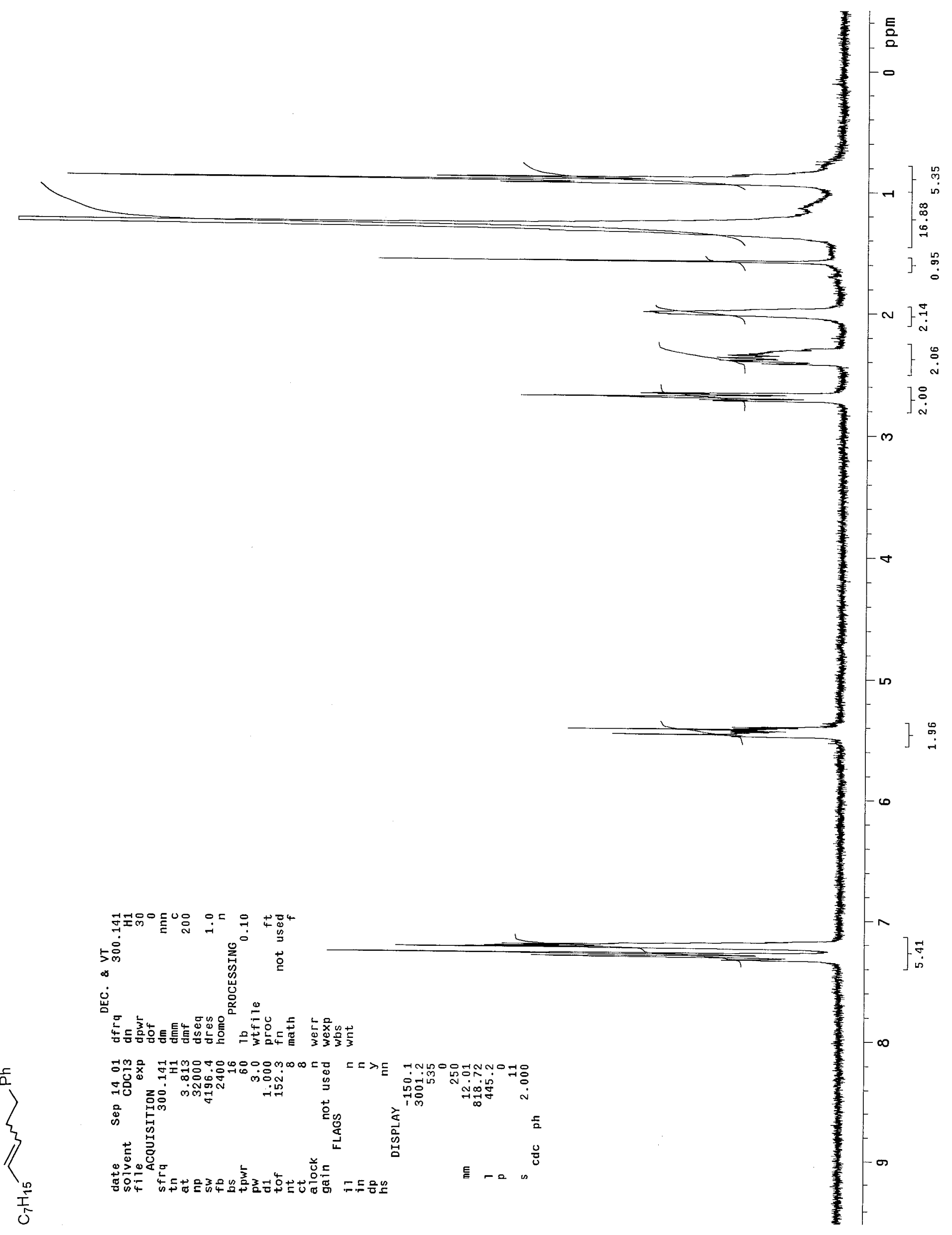




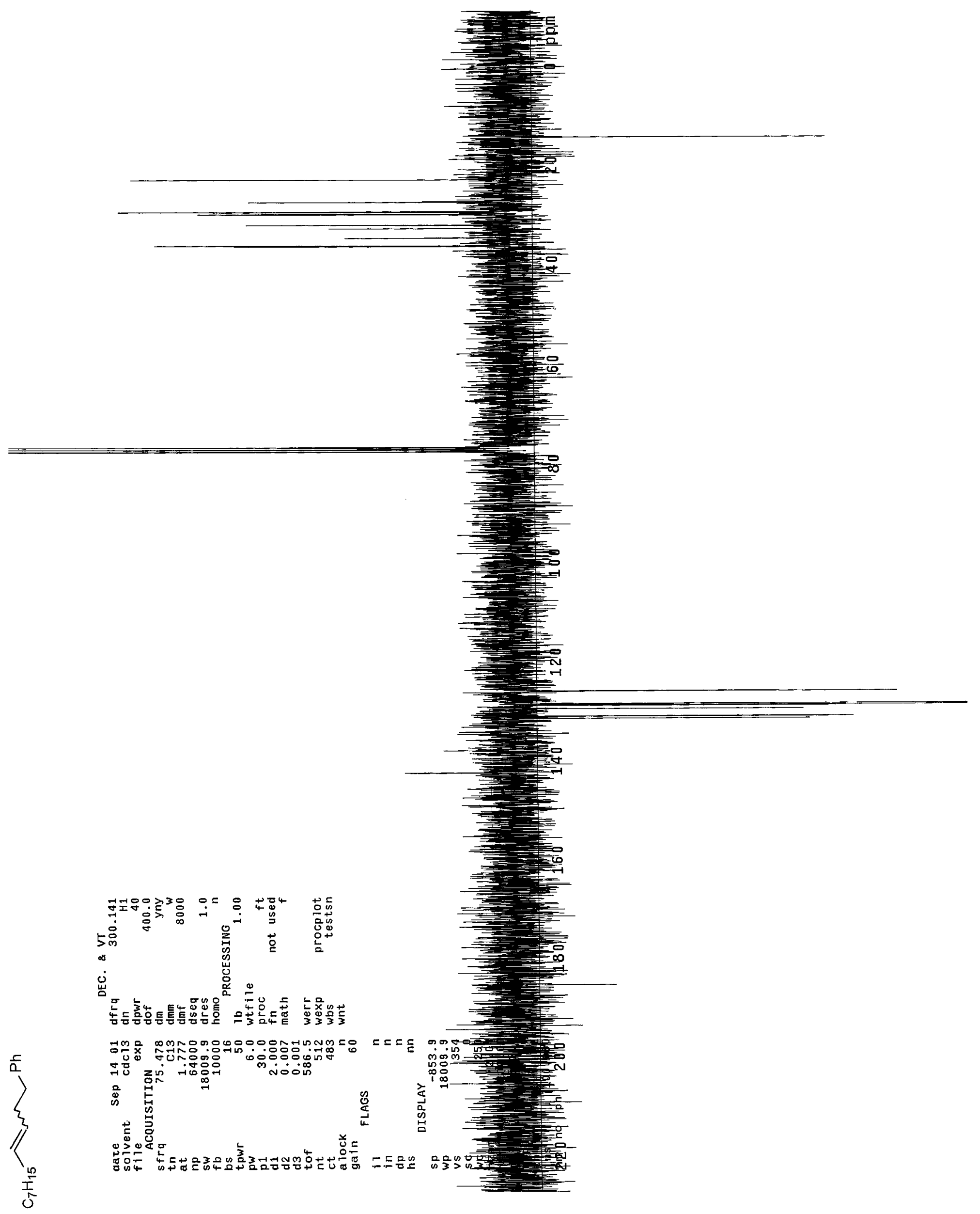




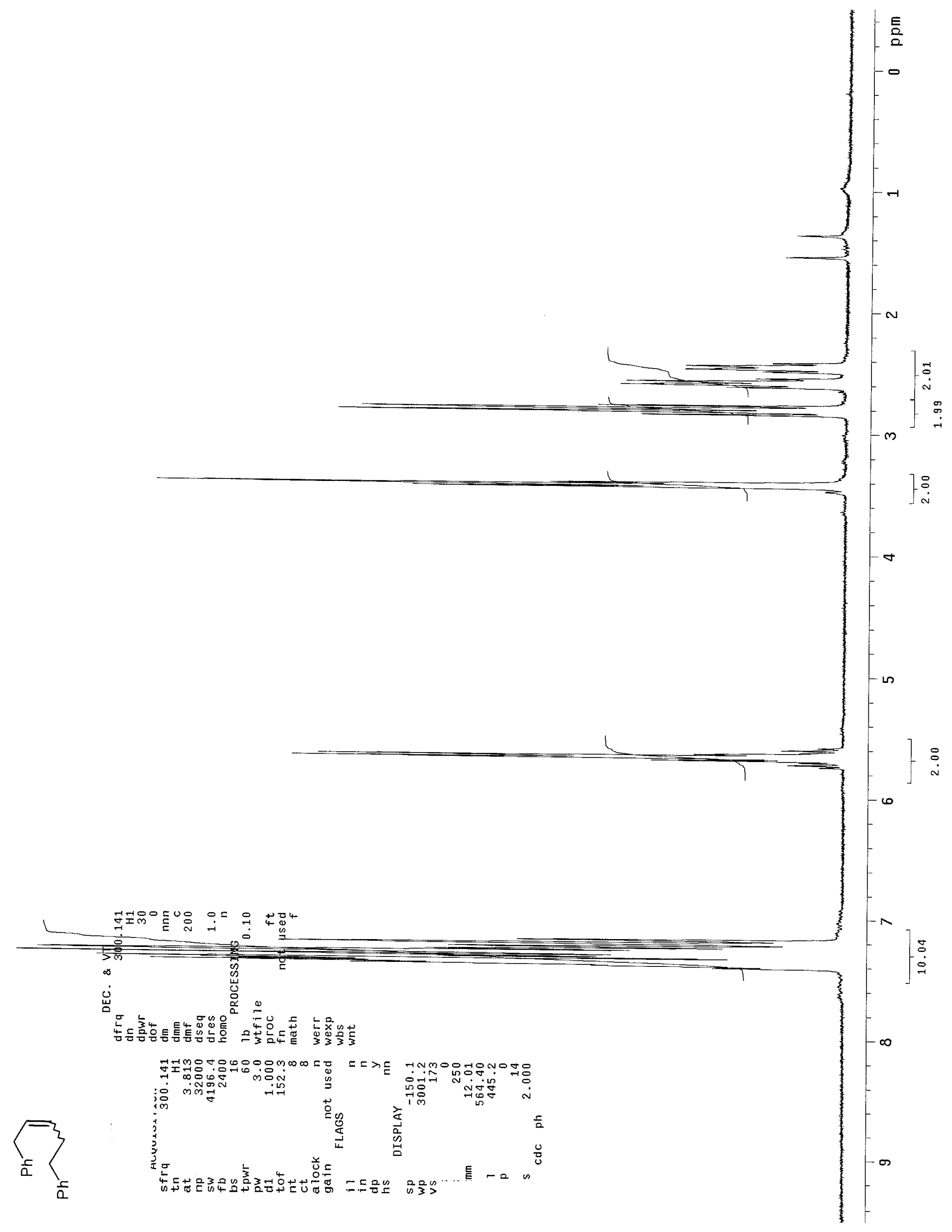




$$
\neq
$$




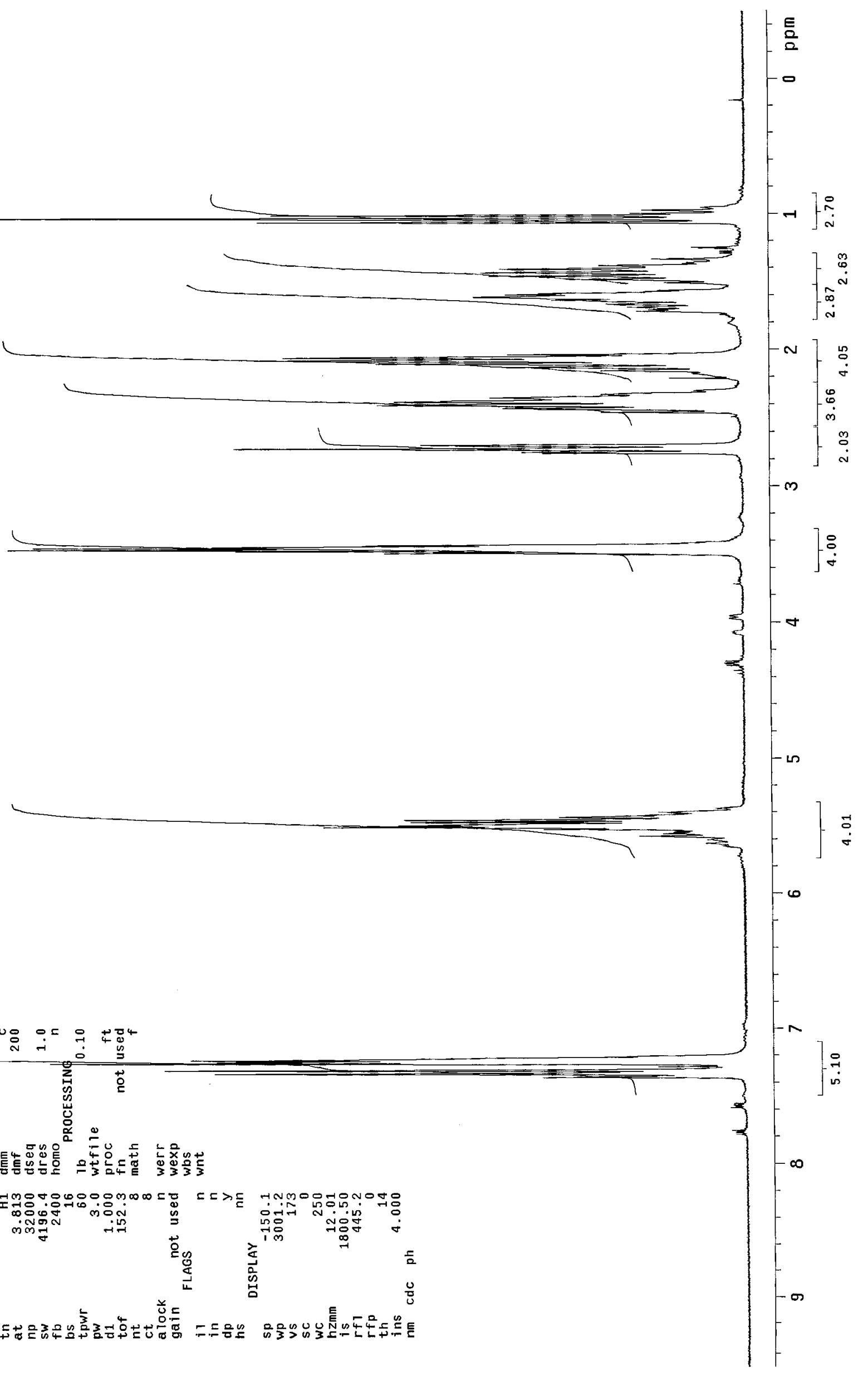




$$
\neq
$$

